\title{
SEMIMARTINGALES AND SUBHARMONIC FUNCTIONS
}

BY

\author{
J. L. DOOB
}

1. Introduction. In the following, the basic measure space will be denoted by $\Omega$, a point of $\Omega$ by $\omega$. The space $\Omega$ will itself have measure 1 , and it will be supposed that the measure is complete. If $x$ is a function on $\Omega$, its value at $\omega_{0}$ will be denoted by $x\left(\omega_{0}\right)$, its integral by $E\{x\}$. The measure of an $\Omega$ set will be denoted by $P\{-\}$ and $\{-\}$ will be used to denote the $\Omega$ set determined by the conditions listed between the braces. A stochastic process $\{x(t), t \in T\}$ is a family of measurable functions (random variables) defined on $\Omega$. The parameter set $T$ will always be linear, and will sometimes contain the point $\infty$.

The conditional expectation of a random variable relative to a set of specified random variables will be denoted by $E\{x \|-\}$, where the specified random variables will be listed after the double bar.

Let $\{x(t), t \in T\}$ be a real stochastic process. Then the process is said to be a semimartingale if, whenever $s<t$, and both values/are in the parameter set,

$$
E\{x(t) \| x(r), r \leqq s\} \geqq x(s)
$$

with probability 1 . We shall suppose throughout this paper that $E\{|x(t)|\}$ is finite, although, for some purposes, it is useful to extend the definition of a semimartingale by weakening this hypothesis.

Let $u$ be a real-valued function defined in a plane domain (open connected set), with

$$
-\infty \leqq u(z)<\infty, \quad u(z) \not \equiv-\infty .
$$

Then $u$ is said to be subharmonic if it is upper semicontinuous, and if, for each $z$ in the domain of definition,

$$
\frac{1}{2 \pi} \int_{0}^{2 \pi} u\left(z+c e^{i \theta}\right) d \theta \geqq u(z),
$$

for sufficiently small $c>0$.

These definitions have formal similarities which suggest that there may be a connection between semimartingales and subharmonic functions which will be fruitful to investigate. This investigation is the purpose of the present paper.

At a superficial level, there is an obvious parallel between semimartingales and subharmonic functions, due to the fact that the basic inequalities (1.1)

Received by the editors February 13, 1953. 
and (1.2) are both inequalities between a function value and an integral average. Thus if the $x(t)$ process is a semimartingale, if $u$ is subharmonic, and if $\phi$ is a continuous convex function, then the stochastic process $\{\phi[x(t)]$, $t \in T\}$ is a semimartingale (if the relevant expectations are finite) and the function $\phi(u)$ is subharmonic. We shall not pursue this formal parallelism further, although it extends surprisingly far, including for example the study of harmonic and subharmonic minorants and their stochastic analogues. We are more interested in a study which brings the two topics together, and thereby obtains new results in the theory of subharmonic and harmonic functions.

A harmonic function is a function $u$ with the property that both $u$ and $-u$ are subharmonic. The probability analogue is a martingale, a stochastic process $\{x(t), t \in T\}$ with the property that both the $x(t)$ and $-x(t)$ processes are semimartingales. Thus there is equality in (1.1) and (1.2) for martingales and harmonic functions respectively. In the martingale case it is useful to allow complex and vector-valued random variables, for which (1.1) (with equality) is then the defining property.

The simplest key to the present study is the trivial remark that, if $z=x+i y$ is a complex random variable, with mutually independent Gaussian real and imaginary parts $x, y$, with

$$
E\{x\}=E\{y\}=0, \quad E\left\{x^{2}\right\}=E\left\{y^{2}\right\},
$$

then, for every positive integer $n, E\left\{z^{n}\right\}=0$. In fact

$$
z^{n}=|z|^{n} e^{i n \theta},
$$

where $|z|, \theta$ are mutually independent random variables, and $\theta$ is uniformly distributed on the interval $[0,2 \pi)$. The assertion is thus indeed trivial. It follows easily that, if $\{z(t), 0 \leqq t<\infty\}$ is a two-dimensional Brownian motion process, defined in $\S 3$, and if $f$ is any polynomial, the stochastic process $\{f[z(t)], 0 \leqq t<\infty\}$ is a martingale. It then follows that this process is a martingale if $f$ is an entire function whose modulus does not become infinite too fast with $|z|$. Going to real parts, corresponding theorems can be deduced for $f$ real and harmonic (when the $f[z(t)]$ process is a martingale) or $f$ real and subharmonic (when the $f[z(t)]$ process is a semimartingale).

Rather than proceeding in this way, we shall prove theorems involving subharmonic functions directly, obtaining theorems for harmonic and regular functions by specialization. The details will be carried through first for subharmonic and harmonic functions in the plane. The $n$-dimensional case $(n>2)$ is somewhat easier, and the corresponding development will be outlined in \$7. No attempt will be made to develop known theorems on subharmonic and harmonic functions by means of probability methods, although such a program has interesting possibilities.

\section{Lemmas on subharmonic functions.}


LemмA 2.1. Let $D_{1}, D_{2}$ be plane domains with a non-null intersection. Let $u_{i}$ be a function defined and subharmonic in $D_{i}$, with $u_{2} \geqq u_{1}$ in $D_{1} \cap D_{2}$. Suppose that, if $z_{0}$ is a boundary point of $D_{2}$ in $D_{1}$, then

$$
u_{1}\left(z_{0}\right)=\limsup _{z \rightarrow z_{0}} u_{2}(z) \quad\left(z \in D_{2}\right) .
$$

Then, if $u^{*}$ is defined by

$$
u^{*}(z)=\left\{\begin{array}{l}
u_{2}(z) \\
u_{1}(z)
\end{array}\right.
$$

$$
\begin{array}{r}
\text { if } z \in D_{2}, \\
\text { if } z \in D_{1}-D_{2},
\end{array}
$$

$u^{*}$ is subharmonic in $D_{1} \cup D_{2}$.

This lemma (which is not new) is a slight variation of the theorem that the maximum of two functions subharmonic in a common domain is subharmonic, and a direct proof is trivial.

In the following we shall describe any plane point set containing all finite points outside some circle as a neighborhood of $\infty$, or, if $\infty$ is included, as an extended neighborhood of $\infty$. Important particular cases are the exterior and extended exterior of a Jordan curve. If a function $u$ is defined in an extended neighborhood of $\infty$, it is said to be harmonic there if it is continuous and if it is harmonic on the set of finite points of the neighborhood.

Lemma 2.2. Let $D$ be a plane domain in the interior of a Jordan curve $C$, and suppose that $C$ is an isolated part of the boundary of $D$. Let $D_{0}$ be a domain whose closure lies in $D$, and let $u$ be a function defined and subharmonic in $D$. Then there is a function $u^{*}$ defined and subharmonic on the union of $D \cup C$ with the exterior of $C$, and a positive constant $c$, such that

(a) $u^{*}(z)=u(z)$ if $z \in D_{0}$,

(b) $u^{*}(z)-c \log |z|$ can be defined at $\infty$ to be harmonic in an extended neighborhood of $\infty$.

We can suppose, enlarging $D_{0}$ if necessary, that $D_{0}$ has as external isolated boundary a Jordan curve $C_{0}$. Let $\Delta$ be a function defined and continuous on $C$ and its exterior, zero on $C_{0}$, harmonic and positive on its exterior, differing by $\log |z|$ from a function harmonic at $\infty$. Such a function can be obtained as follows. If $z_{0} \in D_{0}$, define $\Delta$ as the difference between $\log \left|z-z_{0}\right|$ and the function harmonic in the extended exterior of $C_{0}$ which is the solution of the Dirichlet problem in the extended exterior, with boundary function $\log \left|z-z_{0}\right|$. Since $u$ is upper semicontinuous on $C_{0}$, it is the limit of a monotone decreasing sequence of functions continuous on $C_{0}$. Let $\left\{U_{n}\right\}$ be the corresponding sequence of functions defined and harmonic in the extended exterior of $C_{0}$, solutions of the Dirichlet problem in the extended exterior of $C_{0}$ with these boundary functions on $C_{0}$. The sequence $\left\{U_{n}\right\}$ converges monotonely to a function $U$, the solution of the generalized Dirichlet problem in the extended exterior of $C_{0}$ with boundary function $u$ on $C_{0}$. Let 
$C_{1}$ be a Jordan curve in the interior of $C$, containing $C_{0}$ in its interior. In the following we shall apply Lemma 2.1, and shall match the notation to that lemma's. Let $D_{1}$ be the common part of $D$ and the interior of $C_{1}$, and let $D_{2}$ be the exterior of $C_{0}$. Let $c$ be any positive constant for which

$$
c \inf _{z \in C_{1}} \Delta(z) \geqq \sup _{z \in C_{1}}[u(z)-U(z)],
$$

and define $u_{1}, u_{2}$ by

$$
\begin{array}{ll}
u_{1}(z)=u(z) & \text { if } z \in D_{1}, \\
u_{2}(z)=U(z)+c \Delta(z) & \text { if } z \in D_{2} .
\end{array}
$$

Then Lemma 2.1 states that a function $u^{*}$ exists, as described in the statement of the present lemma, under certain hypotheses. These hypotheses are obviously satisfied here except possibly for the condition we shall now check that $u_{2}(z) \geqq u_{1}(z)$ in $D_{1} \cap D_{2}$, that is, in the annulus between $C_{0}$ and $C_{1}$. The function $u_{1}$ coincides in this annulus with a function $u$ subharmonic in a domain including this annulus and its boundary. The function $u_{2}$ is harmonic in this annulus. Let $u_{2, n}$ be the harmonic function defined like $u_{2}$ but with $U$ replaced by $U_{n}$. Then $u_{2, n}$ is harmonic in the annulus and continuous in the closed annulus. Moreover on the bounding curves of the annulus $u_{2, n} \geqq u_{1}$. Hence, by a fundamental property of subharmonic functions, frequently used as the essential defining property, $u_{2, n}(z) \geqq u_{1}(z)$ in the annulus. When $n \rightarrow \infty$ we find that $u_{2}(z) \geqq u_{1}(z)$ in the annulus, as was to be proved, so that Lemma 2.1 is applicable.

Lemma 2.3. Let $D$ be a plane domain, and let $D_{0}$ be a subdomain whose boundary is bounded and lies in $D$. Let $u$ be a function defined and subharmonic in $D$. Then there is a function w, defined and subharmonic in the finite plane, and a function v, either vanishing identically or of the form

$$
v=-\sum_{j=1}^{n} c_{j} \log \left|z-z_{j}\right|,
$$

$$
c_{j}>0,
$$

such that, if $u^{*}=v+w$ :

(a) $u^{*}(z)=u(z)$ if $z \in D_{0}$;

(b) $w$ is harmonic in a neighborhood of each $z_{j}$ (if $v$ does not vanish identically) and, if $D_{0}$ is not a neighborhood of $\infty$, there is a positive constant $c_{0}$ for which $w-c_{0} \log |z|$ can be defined at $\infty$ to be harmonic in an extended neighborhood of that point;

(c) If $v(z) \not \equiv 0$, each $z_{j}$ is in a bounded component of the complement of $D$.

Note that $u^{*}$ is not subharmonic at the $z_{j}$ 's. The theory is complicated by the fact that a subharmonic function cannot always be extended to be subharmonic on the entire plane, even if the extension is from a subdomain whose closure is a compact subset of the original domain of definition. In 
proving the lemma we can suppose that $D_{0}$ is the domain bounded by a finite number of disjunct Jordan curves $C_{0}, \cdots, C_{n}$, enlarging $D_{0}$ to make this true, if necessary. Here $C_{0}$ is to be the outer bounding curve, unless $D_{0}$ is a neighborhood of $\infty$, in which case $C_{0}$ is to be absent. If $C_{0}$ is present, $C_{1}, \cdots, C_{n}$ may be absent. Further enlarging $D_{0}$, if necessary, we can suppose that the interior of each $C_{j}$ with $j \geqq 1$ contains at least one point not in $D$. (If there is a $C_{j}$ without this property, this $C_{j}$, together with its interior, is added to $D_{0}$.) For each $C_{j}$ with $j \geqq 1$, choose $z_{j}$ in the interior of $C_{j}$, not in $D$. If $C_{0}$ is present, apply Lemma 2.2 to obtain $u^{*}$ defined and subharmonic in the union of the $D_{0} \cup C_{0}$ with the exterior of $C_{0}$, equal to $u$ in $D_{0}$, and differing from a function harmonic in the extended neighborhood of $\infty$ by $c_{0} \log |z|$. To define $u^{*}$ on a $C_{j}$ and its interior when $j \geqq 1$, invert the figure in $z_{j}$ to reduce the problem to the one already solved of extending $u$ outside a curve. Inverting back we find that the extended function $u^{*}$ is defined and subharmonic in the union of $D_{0} \cup C_{j}$ with the interior of $C_{j}$ less the point $z_{j}$, equal to $u$ in $D_{0}$, and differing from a function harmonic in a neighborhood of $z_{j}$ by $-c_{j} \log \left|z-z_{j}\right|$. Repeating this procedure, we define $u^{*}$ everywhere on the plane. Then $v$ is defined by (2.1), if $n>0$, and otherwise $v(z) \equiv 0$, and if $w=u^{*}-v, w$ is defined and subharmonic on the finite plane less the points $z_{1}, \cdots, z_{n}$. If these points are present, we have shown that $w$ can be defined at these points to be harmonic in a neighborhood of each.

3. The Brownian motion process. For each $t \geqq 0$ let $x(t)$ be a vector random variable in $N$ dimensions, with components $x_{1}(t), \cdots, x_{N}(t)$. The stochastic process $\{x(t), 0 \leqq t<\infty\}$ will be called a Brownian motion process if the following conditions are satisfied.

B1. $x_{j}(0, \omega)=0, j \geqq 1$, for almost all $\omega$.

B2. If $0 \leqq t_{0}<\cdots<t_{n}$, the $N n$ random variables

$$
x_{j}\left(t_{k}\right)-x_{j}\left(t_{k-1}\right), \quad k=1, \cdots, n ; j=1, \cdots, N,
$$

are mutually independent.

B3. If $0 \leqq s<t$, the random variable $x_{j}(t)-x_{j}(s)$ is Gaussian, with

$$
E\left\{x_{j}(t)-x_{j}(s)\right\}=0, \quad E\left\{\left[x_{j}(t)-x_{j}(s)\right]^{2}\right\}=\sigma^{2}(t-s) .
$$

Here $\sigma$ is a positive constant, kept fixed throughout this paper.

B4. For almost all $\omega$, the $t$ function $x(\cdot, \omega)$ is continuous, that is almost all sample functions of the $x(t)$ process are continuous. (This condition is very nearly a consequence of $\mathrm{B} 1, \mathrm{~B} 2, \mathrm{~B} 3$.)

Let $\{x(t), 0 \leqq t<\infty\}$ be a Brownian motion process in $N$ dimensions, and let $x_{0}$ be any point of $N$-dimensional space. Then the process $\left\{x_{0}+x(t)\right.$, $0 \leqq t<\infty\}$ will be called a Brownian motion process with initial point $x_{0}$. Let $A$ be a closed set in $N$-dimensional space. Define the $\Omega$ function $\tau$ as the smallest value of $t$ for which $x_{0}+x(t, \omega)$ is a point of $A$, except that $\tau(\omega)=\infty$ if $\omega$ yields a sample Brownian path with $x(0, \omega) \neq 0$, or which is not continu- 
ous, or which is continuous but never meets $A$. That is, $\tau$ is the time to the first meeting with $A$ of a continuous Brownian path starting from $x_{0}$. Then it is easily verified that $\tau$ is a measurable $\Omega$ function. We give the verification because a fact it implies will be used below. Let $A_{n m}$ be the set of points at distance $\leqq 1 / n$ from $A$ and at distance $\leqq m$ from $x_{0}$. Then

$$
\{\tau(\omega) \leqq s\}=\bigcup_{m} \bigcap_{n} \bigcup_{r<s}\left\{x_{0}+x(r, \omega) \in A_{n m}\right\}
$$

neglecting the $\Omega$ set $\{x(0, \omega) \neq 0\}$, and that corresponding to discontinuous Brownian paths. It follows that $\tau$ is measurable. Define the stochastic process $\left\{x^{*}(t), 0 \leqq t<\infty\right\}$ by

$$
x^{*}(t, \omega)= \begin{cases}x(t, \omega) & \text { if } t<\tau(\omega), \\ x[\tau(\omega), \omega] & \text { if } t \geqq \tau(\omega) .\end{cases}
$$

Then the $x^{*}(t)$ process will be called a Brownian motion process from $x_{0}$, stopped on $A$. In particular, if $\tau(\omega)<\infty$ with probability 1 , we shall add $\infty$ to the parameter set of the process, setting

$$
x^{*}(\infty, \omega)=x[\tau(\omega), \omega] \quad \text { if } \tau(\omega)<\infty,
$$

in agreement with the definition for $t<\infty$. Define $x^{*}(\infty, \omega)$ arbitrarily where $\tau(\omega)=\infty$. It is essential to the application of stopped processes that, as is obvious from the above, the condition $\tau(\omega) \leqq s$ is a condition on the sample functions at values of the argument $\leqq s$, so that the $\Omega$ set $\{\tau(\omega) \leqq s\}$ is independent of the class of differences $\{x(t)-x(s), t>s\}$.

The above remarks go through with obvious changes if the initial point $x_{0}$ is a random variable which is not identically constant and which is independent of the class of random variables $\{x(t), 0 \leqq t<\infty\}$. Under this hypothesis on $x_{0}$, if it is not identically constant, with the above conventions and notation, and if $\tau<\infty$ with probability 1 , define

$$
\begin{aligned}
y_{0}(\omega) & =x^{*}(\infty, \omega), & \\
y(t, \omega) & =x[\tau(\omega)+t, \omega]-x^{*}(\infty, \omega), & \text { if } t \geqq 0 .
\end{aligned}
$$

Then it can be shown that the $y(t)$ process is a Brownian motion process, with the same variance parameter $\sigma^{2}$ as the $x(t)$ process, and that $y_{0}$ is independent of the class of random variables $\{y(t), 0 \leqq t<\infty\}$. Thus, if the initial position of a Brownian motion is independent of the later motion, and if the motion is certain to meet a barrier, $A$, then, counting time from the meeting, the motion continues as a new Brownian motion, with, of course, a new initial distribution confined to $A$, the new initial position being independent of the later motion. Although this fact gives considerable insight into some of the results of this paper, it will not be proved, because it is intuitively obvious, and because it is a special case of a much more general theorem which will be proved elsewhere. 
Throughout this paper, we shall consider Brownian motion processes with constant initial points. The generalization of the results to randomized initial positions will be obvious.

From now on, except in $\$ 7$, we shall consider only Brownian motions in two dimensions. In that case we write $z(t)=x_{1}(t)+i x_{2}(t)$, so that the defining variables of the process are complex-valued instead of vector-valued.

4. Semimartingales defined by subharmonic functions. In this section we show the connection between semimartingales and subharmonic functions, the latter defined in plane domains, basing the treatment on the rather superficial Theorem 4.1. For proofs of the facts on semimartingales and martingales used below, see [7].

Several of the theorems cover simultaneously real subharmonic and harmonic functions, as well as complex regular (analytic) functions. The specialization from subharmonic to harmonic and regular functions will always be trivial.

It is useful to specialize the martingale definition of $\$ 1$ slightly. Let $T$ be a linear set and suppose that, for each $t \in T, \mathcal{F}(t)$ is a Borel field of measurable $\Omega$ sets such that

(a) $\mathcal{F}(s) \subset \mathcal{F}(t)$ if $s<t$;

(b) $x(t)$ is a random variable measurable relative to $\mathcal{f}(t)$, or at least equal almost everywhere to an $\Omega$ function that is;

(c) $E\{x(t) \| \mathcal{F}(s)\} \geqq x(s)$ if $s<t$ with probability 1 , where the exceptional set may depend on $s, t$. (See [7] for a discussion of conditional expectations relative to a field.)

If these three conditions are satisfied, the $x(t)$ process is called a semimartingale relative to the specified family of Borel fields, and is written $\{x(t), \mathcal{f}(t), t \in T\}$. The process is then necessarily a semimartingale in the sense of the definition of $\$ 1$, and conversely if the $x(t)$ process is a semimartingale in the sense of that definition, it is a semimartingale relative to a class of Borel fields. In fact, $\mathcal{F}(t)$ can be taken as the smallest Borel field of $\Omega$ sets relative to which every $x(s)$ with $s \leqq t$ is measurable.

THEOREM 4.1. Let $u$ be subharmonic [harmonic or regular] in the finite plane, and suppose that, for every positive $c$,

$$
\int_{0}^{2 \pi} d \theta \int_{0}^{\infty}\left|u\left(r e^{i \theta}\right)\right| e^{-c r^{2}} r d r<\infty .
$$

Let $z_{0}$ be any point of the plane, and let $\{z(t), 0 \leqq t<\infty\}$ be a Brownian motion process starting from $z_{0}$. Then the stochastic process

$$
\{u[z(t)], 0<t<\infty\}
$$

is a semimartingale [martingale]. If $u\left(z_{0}\right)>-\infty$, the conclusion holds with the enlarged parameter interval $[0, \infty)$. 
The hypothesis (4.1) could be weakened somewhat if semimartingales with non-absolutely integrable random variables were allowed, but we omit this generalization because it would not bring advantages in later developments. According to this theorem, if $R$ is any polynomial, the first two of the following processes are semimartingales, and the third is a martingale:

$$
\begin{gathered}
\{\log |R[z(t)]|, 0<t<\infty\}, \quad\{|R[z(t)]|, 0 \leqq t<\infty\}, \\
\{R[z(t)], 0 \leqq t<\infty\} .
\end{gathered}
$$

The first process is a semimartingale on $[0, \infty)$ if $R\left(z_{0}\right) \neq 0$.

Let $\mathcal{F}(s)$ be the smallest Borel field of $\Omega$ sets containing all sets of probability 0 , and such that every $z(t)$ with $t \leqq s$ is measurable relative to $\mathcal{f}(s)$. Then we shall prove a slightly stronger result than that stated in the theorem in that we shall prove that the $u[z(t)]$ process is a semimartingale or martingale relative to the $\mathcal{F}(s)$ family of fields. According to (4.1), if $t>0$ and if $z=r e^{i \theta}$,

$$
\begin{aligned}
E\{|u[z(t)]|\} & =\frac{1}{2 \pi t \sigma^{2}} \int_{0}^{2 \pi} d \theta \int_{0}^{\infty} e^{-\left|z-z_{0}\right|^{2} / 2 t \sigma^{2}}\left|u\left(r e^{i \theta}\right)\right| r d r \\
& \leqq \frac{1}{c} \int_{0}^{2 \pi} d \theta \int_{0}^{\infty} e^{-c r^{2}}\left|u\left(r e^{i \theta}\right)\right| r d r<\infty,
\end{aligned}
$$

for some positive constant $c$. If $u$ is subharmonic, and if $0<s \leqq t$,

$$
\begin{aligned}
E\{u[z(t)] \| \mathcal{F}(s)\} & =E\{u[z(t)] \| z(s)\} \\
& =\frac{1}{2 \pi(t-s) \sigma^{2}} \int_{0}^{2 \pi} d \theta \int_{0}^{\infty} e^{-\rho^{2} / 2(t-s) \sigma^{2}} u\left[\rho e^{i \theta}+z(s)\right] \rho d \rho \\
& \geqq \frac{1}{(t-s) \sigma^{2}} \int_{0}^{\infty} e^{-\rho^{2} / 2(t-s) \sigma^{2}} u[z(s)] \rho d \rho=u[z(s)]
\end{aligned}
$$

with probability 1 . Thus the $u[z(t)]$ process is a semimartingale relative to the specified Borel fields. If the function $u$ is harmonic or regular, this inequality becomes an equality, so that in either of these cases the $u[z(t)]$ process is a martingale. If $u\left(z_{0}\right)>-\infty, u[z(0)]=u\left(z_{0}\right)$ is finite, and the restriction of positivity of parameter values becomes unnecessary.

As an example of the application of this theorem we prove the following corollary due to Lévy [13], which will be strengthened considerably below.

COROLlary If $z_{0}, z_{1}$ are arbitrary points of the plane (equality not excluded) the probability is 0 that a Brownian path starting from $z_{0}$ ever passes through $z_{1}$, for $t>0$.

In fact the function $u$ defined by

$$
u=\log \left|z-z_{0}\right|+\log \left|z-z_{1}\right|
$$

is subharmonic in the plane, and obviously satisfies the condition (4.1). Hence 
the $u[z(t)]$ stochastic process of Theorem 4.1 with this choice of $u$ is a semimartingale. Now almost all the sample functions of a semimartingale are bounded on the rational points of every closed interval of parameter points. It follows that almost all sample functions of the $u[z(t)]$ process are bounded on the rational points of every interval $[\delta, 1 / \delta]$ with $\delta>0$. Hence almost no Brownian path from $z_{0}$ passes through $z_{1}$ for positive $t$. The restriction to rational parameter values in this argument is unnecessary here, but was made to avoid unnecessary remarks on separability of stochastic processes.

In the following we shall frequently deal with a Brownian motion process $\{z(t), 0 \leqq t<\infty\}$ starting from a point $z_{0}$, the process $\left\{z^{*}(t), 0 \leqq t<\infty\right\}$ obtained by stopping the given process on some closed set, and the process $\left\{u\left[z^{*}(t)\right], 0 \leqq t<\infty\right\}$, where $u$ is a specified function of a complex variable defined on the finite plane. The $z(t)$ process is a martingale, according to Theorem 4.1. (Of course this assertion has a trivial direct proof.) The $z(t)$ process has continuous sample functions, with probability 1 , and the $z^{*}(t)$ process is obtained from the $z(t)$ process by a method called optional stopping. (See [7] for a detailed discussion of optional stopping, and proofs of its properties used below.) Since semimartingales and martingales remain semimartingales and martingales under optional stopping, the $z^{*}(t)$ process is also a martingale, specifically a martingale relative to a family of Borel fields defined in terms of the closed stopping set and the $\mathcal{f}(t)$ family used in the proof of Theorem 4.1. According to this same reasoning, if the $u[z(t)]$ process has continuous sample functions with probability 1 , and is a semimartingale [martingale], the $u\left[z^{*}(t)\right]$ process, which can also be considered as obtained from the $u[z(t)]$ process by optional stopping, is a semimartingale [martingale], relative to the same family of Borel fields as the $z^{*}(t)$ process. As usual, the field family is the key to the situation. The fact that this family does not depend on the choice of $u$ is important. For example, we shall need the fact that for two functions $u_{1}$ and $u_{2}$ yielding semimartingales, $u_{1}+u_{2}$ also yields a semimartingale, and the sum of two semimartingales is not necessarily a semimartingale unless the summands are semimartingales relative to the same family of fields.

Lemma 4.2. Let $D$ be a plane domain, with boundary C. Suppose that $z_{0} \in D$, and that $z_{1}$ is not in the closure of $D$. Then if $\left\{z^{*}(t), 0 \leqq t<\infty\right\}$ is the Brownian motion process from $z_{0}$, stopped when it meets $C$, the process

$$
\left\{\log \left|z^{*}(t)-z_{1}\right|, 0 \leqq t<\infty\right\}
$$

is a martingale.

To simplify the notation, we suppose in the following that $z_{1}=0$. Let $\{z(t), 0 \leqq t<\infty\}$ be the Brownian motion process starting at $z_{0}$. By Theorem 4.1 the $\log |z(t)|$ process is a semimartingale on $[0, \infty)$. It follows that the $\log \left|z^{*}(t)\right|$ process, which is obtained from the $\log |z(t)|$ process by optional 
stopping, is also a semimartingale. Hence, according to the defining inequality of semimartingales

$$
\log \left|z_{0}\right|=E\left\{\log \left|z^{*}(0)\right|\right\} \leqq E\left\{\log \left|z^{*}(t)\right|\right\}
$$

To prove that the $\log \left|z^{*}(t)\right|$ process is a martingale, we must prove that there is equality here. In proving this lemma, we can assume that $C$ is a circle with center $z_{1}=0$. In fact if $C$ is not such a circle, let $C^{\prime}$ be such a circle, which, together with its interior, lies outside of $D$. If the lemma is true for $C$ replaced by $C^{\prime}$, that is, if the $\log |z(t)|$ process stopped when the Brownian motion meets $C^{\prime}$ is a martingale, then that stopped still earlier, when the motion meets $C$, is also a martingale, since stopped martingales are martingales. Thus we can and shall suppose that $C=C_{r}$ is a circle of radius $r$, with the origin as center. Let $C_{R}$ be a circle of radius $R$ with the same center, where

$$
r<\left|z_{0}\right|=\rho<R .
$$

Let $\left\{z_{R}(t), 0 \leqq t \leqq \infty\right\}$ be the Brownian motion process starting from $z_{0}$ and stopped on $C_{R} \cup C_{r}$. The reasoning used above shows that the log $\left|z_{R}(t)\right|$ process is a semimartingale. Hence

$$
\log \left|z_{0}\right|=E\left\{\log \left|z_{R}(0)\right|\right\} \leqq E\left\{\log \left|z_{R}(t)\right|\right\},
$$$$
0 \leqq t<\infty,
$$

and the right side of this inequality is monotone nondecreasing in the variable t. Now.

$$
\lim _{t \rightarrow \infty} \log \left|z_{R}(t)\right|=\log \left|z_{R}(\infty)\right|,
$$

with probability 1 , and these random variables are uniformly bounded. Hence

$$
\log \left|z_{0}\right| \leqq E\left\{\log \left|z_{R}(\infty)\right|\right\},
$$

and there is equality in (4.3) for all $t$ if there is equality in (4.4). Let $p$ be the probability that a Brownian path from $z_{0}$ meets $C_{R}$ before it meets $C_{r}$. Then (4.4) can be written in the form

$$
\log \left|z_{0}\right| \leqq p \log R+(1-p) \log r
$$

that is,

$$
p \geqq \frac{\log (\rho / r)}{\log (R / r)} .
$$

Now suppose that there is equality in $\left(4.5^{\prime}\right)$. Then we shall show that it follows that there is equality in (4.2), and we have seen that the latter equality implies the truth of the lemma. In fact, if there is equality in $\left(4.5^{\prime}\right)$, there is also equality in (4.5) and (4.4), and hence there is equality in (4.3) for all $t$. To prove that there is then equality in (4.2), we let $R \rightarrow \infty$. Then 


$$
\lim _{R \rightarrow \infty} \log \left|z_{R}(t)\right|=\log \left|z^{*}(t)\right|
$$

with probability 1 , so that it is sufficient to prove that integration to the limit is admissible. Now

$$
|\log | z_{R}(t)|| \leqq|\log r|+\frac{1}{r}\left[\max _{0 \leqq s \leqq t}\left|z(s)-z_{0}\right|+\left|z_{0}\right|\right] .
$$

The right side of this inequality is independent of $R$, and has a finite expectation. (According to the classical results of Bachelier, the maximum displacement of a one-dimensional Brownian motion over $[0, t]$ has the same distribution as the absolute value of the displacement at $t$.) Hence integration to the limit in (4.6) is justified, as was to be proved. There remains, however, the proof that there is equality in $\left(4.5^{\prime}\right)$, which we now see is the key to the lemma. Now $\left(4.5^{\prime}\right)$ with equality is a very special case of a theorem of Kakutani [11]. Since this special case is so simple, and since Kakutani has not yet published the details of his proof, the proof of this special case will be outlined for completeness. The probability $p$ depends only on $\rho, r, R$, because the situation is circularly symmetric in the origin. This probability is unaffected by a transformation $t^{\prime}=c t$ with $c>0$ in the time parameter. Since the $z(t)$ process and the $z(c t) / c^{1 / 2}$ process have the same distributions, $p$ is unaffected by a similarity of the plane in the origin, that is, $p$ is a function of $\rho / r, R / r$. If $\rho<R_{1}<R$, a path from $z_{0}$ must meet the circle of radius $R_{1}$ with the origin as center before it meets $C_{R}$. Hence $p$ is monotone nonincreasing in $R$ for fixed $\rho, r$. Similarly it is monotone nonincreasing in $r$ for fixed $\rho, R$. Now if $c>1$ an increase of $\rho$ to $c \rho$ has the same effect on $p$ as a simultaneous decrease of $r$ to $r / c$ and of $R$ to $R / c$. Hence this change of $\rho$ can only increase $p$. From now on let $r, R$ be fixed. Then $p$ is a monotone nondecreasing function of $\rho$. A Brownian motion from $z$ meets a circle centered at $z$ in a point uniformly distributed on this circle. The Brownian motion from there on can be considered as a new motion with initial value a random variable independent of the later motion. Then $p=p(z)$ is equal to its average over small circles with center $z$, so that it is also equal to its average over small discs with center $z$. Since the latter average defines a continuous function of $z$, for a fixed disc radius, $p$ is continuous. Then the above average property means that $p$ is harmonic. Moreover $p$ takes on the boundary value 1 on $C_{R}$ and 0 on $C_{r}$. In fact, as Lévy has remarked, a plane Brownian motion circles around its initial point in arbitrarily small time intervals, so that if the motion starts near a circle it meets the circle soon. (The fact that the boundary value is 1 on $C_{R}$ also follows from $\left(4.5^{\prime}\right)$.) Since the right side of $\left(4.5^{\prime}\right)$ is the only harmonic function defined in the annulus bounded by $C_{r}$ and $C_{R}$ with the specified boundary values, there must be equality in $\left(4.5^{\prime}\right)$, as was to be proved.

This furnishes the proof of Lemma 4.2. Note that we have not proved that 
the stochastic process of the lemma is a martingale over the augmented parameter interval $[0, \infty]$, and in fact it is not, because there is obviously not equality in (4.2) when $t=\infty$.

We have used in this proof the fact that a Brownian path from $z_{0}$ is certain to meet $C_{R} \cup C_{r}$. This fact follows from the trivial fact that, for any bounded set $A$, the probability that a Brownian path from $z_{0}$ lies in $A$ at time $t$ approaches 0 when $t \rightarrow \infty$. Thus almost every Brownian path from $z_{0}$ is an unbounded continuous curve. More generally, $\left(4.5^{\prime}\right)$, which we have now proved is true with equality, implies, when $R \rightarrow \infty$, that almost every Brownian path from $z_{0}$ meets $C_{r}$. Since $C_{r}$ is any circle not containing $z_{0}$, almost every Brownian path from $z_{0}$ is a continuous curve dense in the plane. This result, which we state as a corollary for convenient reference, is due to Lévy [13].

Corollary. Almost every Brownian path from a point is an everywhere dense continuous curve.

Let $u$ be a function defined and subharmonic in a plane domain $D$. Such a function is not necessarily continuous, even if finite-valued. Let $z_{0}$ be a point of $D$. Then we shall say that $u$ is continuous on almost all Brownian paths from $z_{0}$ if, when $\{z(t), 0 \leqq t<\infty\}$ is a Brownian motion process starting from $z_{0}, u[z(t, \omega)]$ defines, for almost all $\omega$, a finite-valued continuous function of $t$ for $t$ less than the first value of the parameter, if there is one, for which the path meets the boundary of $D$, except that, if $u\left(z_{0}\right)=-\infty$, we consider only $t>0$. It will be proved below that, for each triple $u, D, z_{0}, u$ is continuous on almost all Brownian paths from $z_{0}$. In particular, if $u$ is harmonic or regular, it is continuous and there is nothing to prove. We shall make the assertion of continuity on Brownian paths a hypothesis, where needed in this section, since its proof would interrupt the logic of the discussion at this time.

Theorem 4.3. Let $D$ be a plane domain, and let $D_{0}$ be a subdomain, with a bounded boundary $C_{0} \subset D$. Let $z_{0} \in D_{0}$, and let $\left\{z^{*}(t), 0 \leqq t \leqq \infty\right\}$ be a Brownian motion process starting from $z_{0}$ and stopped on $C_{0}$. Let $u$ be defined and subharmonic [harmonic or regular] in $D$. If $u$ is subharmonic it is supposed continuous on almost all Brownian paths starting from $z_{0}$. If $D_{0}$ is a neighborhood of $\infty$, it is supposed that, for some $K>0$, and for every $c>0$,

$$
\int_{0}^{2 \pi} d \theta \int_{K}^{\infty}\left|u\left(r e^{i \theta}\right)\right| e^{-c r^{2}} r d r<\infty .
$$

Then the process $\left\{u\left[z^{*}(t)\right], 0<t<\infty\right\}$ is a semimartingale [martingale]. In this assertion, the point $t=0$ can be added to the parameter interval if $u\left(z_{0}\right)>-\infty$, and both $t=0$ and $t=\infty$ can be added to the parameter interval if $u$ is bounded in $D_{0}$.

Note that Lemma 4.2 is a special case of this theorem. This special case 
is important because through it and Lemma 2.3 the general case can be reduced to an application of Theorem 4.1.

We shall consider only the case of subharmonic $u$, since the other cases can be trivially reduced to this one. It is legitimate to discuss $z^{*}(\infty)$, since, according to the corollary to Lemma 4.2, almost every Brownian path from $z_{0}$ meets $C_{0}$. We shall suppose first that $u\left(z_{0}\right)>-\infty$. Let $u^{*}, v, w$ be the functions defined in Lemma 2.3. Then $w$ is continuous on the complement of a slightly larger domain than $D_{0}$, so that $w$ is continuous on almost all Brownian paths starting from $z_{0}$, not only in $D$ but in the whole plane. Since $w$ is subharmonic in the finite plane and satisfies the analytic condition of Theorem 4.1 , the stochastic process $\{w[z(t)], 0 \leqq t<\infty\}$ (where the $z(t)$ process is the Brownian motion process starting from $z_{0}$ ) is a semimartingale. Then the process obtained by stopping this process when the Brownian motion meets $C_{0}$, that is the $w\left[z^{*}(t)\right]$ process, is also a semimartingale. According to Lemma 4.2 , the process $\left\{v\left[z^{*}(t)\right], 0 \leqq t<\infty\right\}$ is a martingale. Since these processes are semimartingales or martingales relative to the same family of Borel fields (see the discussion at the beginning of this section) it follows that the sum process

$$
\left\{u^{*}\left[z^{*}(t)\right], 0 \leqq t<\infty\right\}=\left\{u\left[z^{*}(t)\right], 0 \leqq t<\infty\right\}
$$

is a semimartingale. Moreover, because of the continuity on Brownian paths,

$$
\lim _{t \rightarrow \infty} u\left[z^{*}(t)\right]=u\left[z^{*}(\infty)\right]
$$

with probability 1 . Since the semimartingale property can be defined in terms of inequalities between integrals of the random variables of the process on certain sets, it follows that, if $u$ is bounded in $D_{0}$, the $u\left[z^{*}(t)\right]$ process is a semimartingale on the interval $[0, \infty]$. Finally suppose that $u\left(z_{0}\right)=-\infty$. To finish the proof we must show that the process $\left\{u\left[z^{*}(t)\right], 0<t<\infty\right\}$ is a semimartingale. The reasoning used above is no longer valid, because one condition that a semimartingale remain one after optional stopping is that the expectations of the semimartingale random variables be bounded from below. In the present case $E\{u[z(t)]\}$ is bounded from below by $E\{u[z(0)]\}$ $=u\left(z_{0}\right)$, and the latter quantity is no longer finite. Let $G_{n}$ be a disc, with center $z_{0}$ and radius $1 / n$, where $n$ is so large that $G_{n} \subset D_{0}$. Let $u_{n}$ be the function equal to $u$ in $D-G_{n}$ and equal in $G_{n}$ to the function harmonic in $G_{n}$ which is a solution of the Dirichlet problem in $G_{n}$ with boundary function $u$. By a standard subharmonic function argument, $u_{n}$ is subharmonic in $D$ and . $u \leqq u_{n+1} \leqq u_{n}$. By what we have already proved, since $u_{n}\left(z_{0}\right)$ is finite, the process

$$
\left\{u_{n}\left[z^{*}(t)\right], 0 \leqq t<\infty\right\}
$$

is a semimartingale, and in particular this means that the random variables of the process have finite expectations. If $\tau$ is the first value of $t$ at which 
a Brownian path from $z_{0}$ meets $C_{0}$,

$$
\begin{aligned}
E\left\{\left|u\left[z^{*}(t)\right]\right|\right\} & =\int_{\{\tau(\omega) \geqq t\}}|u[z(t)]| d P+\int_{\{\tau(\omega)<t\}}\left|u\left[z^{*}(t)\right]\right| d P \\
& =\int_{\{\tau(\omega) \geqq t\}}\left|u^{*}[z(t)]\right| d P+\int_{\{\tau(\omega)<t\}}\left|u_{n}\left[z^{*}(t)\right]\right| d P \\
& \leqq E\left\{\left|u^{*}[z(t)]\right|\right\}+E\left\{\left|u_{n}\left[z^{*}(t)\right]\right|\right\}<\infty, \quad t>0 .
\end{aligned}
$$

Thus $u_{n}\left[z^{*}(t)\right]$ decreases to $u\left[z^{*}(t)\right]$ for positive finite $t$, all these random variables are integrable, and the $u_{n}\left[z^{*}(t)\right]$ process is a semimartingale relative to a family of Borel fields not depending on $n$. Since the semimartingale property can be expressed in terms of inequalities between integrals of process random variables on certain sets of the specified family of Borel fields, when $n \rightarrow \infty$ we find that the $u\left[z^{*}(t)\right]$ process is also a semimartingale relative to this same family, for $t>0$.

Corollary. Let $D, D_{0}, C_{0}, z_{0}, z^{*}(t)$ be as in Theorem 4.3. Let $u$ be defined and subharmonic in $D$, and harmonic in $D_{0}$, continuous on almost all Brownian paths starting at $z_{0}$. If $D_{0}$ is a neighborhood of $\infty$, it is supposed that $u$ satisfies the analytic condition of Theorem 4.3. Then, if $u$ is bounded in a neighborhood of $C_{0}$ in $D_{0}$, the process $\left\{u\left[z^{*}(t)\right], 0 \leqq t<\infty\right\}$ is a martingale, and the point $t=\infty$ can be added to the parameter set in this assertion if $u$ is bounded in $D_{0}$.

According to Theorem 4.3, the $u\left[z^{*}(t)\right]$ process is a semimartingale on the parameter interval $[0, \infty)$. Then $E\left\{u\left[z^{*}(t)\right]\right\}$ defines a monotone nondecreasing function of $t$, so that

$$
u\left(z_{0}\right)=E\left\{u\left[z^{*}(0)\right]\right\} \leqq E\left\{u\left[z^{*}(t)\right]\right\},
$$

and the $u\left[z^{*}(t)\right]$ process is a martingale if and only if there is equality in (4.7). For each $n \geqq 1$ let $D_{n}$ be a domain containing $z_{0}$, whose closure lies in $D_{0}$, with a bounded boundary, and satisfying the relations

$$
D_{1} \subset D_{2} \subset \cdots, \bigcup_{1}^{\infty} D_{n}=D_{0} \text {. }
$$

We can also suppose, since $u$ is bounded in a neighborhood of $C_{0}$ in $D_{0}$, that, for some constant $L,|u(z)| \leqq L$, for $z$ on the boundary of each $D_{n}$. Let $\left\{z_{n}^{*}(t), 0 \leqq t \leqq \infty\right\}$ be the given Brownian motion process starting from $z_{0}$, stopped on the boundary of $D_{n}$. According to Theorem 4.3 , the $u\left[z_{n}^{*}(t)\right]$ process is a martingale on $[0, \infty)$, so that

$$
u\left(z_{0}\right)=E\left\{u\left[z_{n}^{*}(t)\right]\right\}, \quad 0 \leqq t<\infty .
$$

To prove the first statement of the corollary, we let $n \rightarrow \infty$ in (4.8), to obtain equality in (4.7). In fact, 


$$
\lim _{n \rightarrow \infty} u\left[z_{n}^{*}(t)\right]=u\left[z^{*}(t)\right]
$$

with probability 1 , in view of the hypothesis of the continuity of $u$ on almost all Brownian paths from $z_{0}$, and we now justify integration to the limit in (4.8). If $u$ is bounded in $D_{0}$, this justification is a triviality, and the above argument is valid for $t \leqq \infty$. Under the weaker hypothesis of the first part of the corollary, the following argument can be used. The random variable (fixed $t$ )

$$
w_{n}=\left|u\left[z_{n}^{*}(t)\right]-u\left[z^{*}(t)\right]\right|
$$

goes to 0 with probability 1 when $n \rightarrow \infty$, and is dominated by $L$ $+\left|u\left[z^{*}(t)\right]\right|$, so that $E\left\{w_{n}\right\} \rightarrow 0$, as was to be proved.

5. Brownian motion and logarithmic capacity. Let $C_{R}$ be a circle (perimeter) in the complex plane, of radius $R$, center the origin, interior $D_{R}$. Let $A$ be a compact subset of $D_{R}$, and let $\mu$ be a finite-valued measure of Borel subsets of $A$. We shall discuss in this section the potential induced by the charge $\mu$ distributed over $A$, relative to $C_{R}$, that is, the function $v$ defined by

$$
v(z)=\int_{A} \log \left|\frac{R^{2}-\bar{z} w}{R(z-w)}\right| \mu(d w),
$$$$
|z|<R .
$$

Here $v \geqq 0,-v$ is subharmonic in $D_{R}$, harmonic in $D_{R}-A$, with boundary value 0 on $C_{R}$. Moreover $v$ can be extended to be harmonic in an annulus enclosing $C_{R}$, and then $-v$ can be extended, using Lemma 2.2 , to be subharmonic in the finite plane, in such a way that the extended function is continuous in the complement of $A$ and is logarithmically infinite at $\infty$.

If there is no $\mu$ for which $v$ is a bounded function in $D_{R}, A$ is said to have zero capacity relative to $C_{R}$.

The concepts to be defined in this paragraph will all be relative to $C_{R}$, and this qualifying phrase will be omitted. See [5] for a discussion of the potential theoretic concepts described. If $A \subset D_{R}$, and if $A$ is compact, and not of zero capacity, there is a $\mu$ for which $v(z)=1$ on $A$ except possibly on the union of a sequence of compact sets of zero capacity. This measure is called the equilibrium distribution of $A, \mu(A)$ is called the capacity of $A$, and the corresponding $v$ is called the equilibrium potential of $A$. The equilibrium potential $v$ is lower semicontinuous in $D_{R}, 0 \leqq v \leqq 1$, and $v$ is continuous at every point $z$ of $A$ where $v(z)=1$. If $A \subset D_{R}$ but if $A$ is otherwise arbitrary, its interior capacity is defined as the upper limit of the capacities of its compact subsets, and its exterior capacity is defined as the lower limit of the interior capacities of the open sets which contain it. If $A$ is open or closed its interior and exterior capacities are equal. The union of a sequence of compact sets of exterior capacity 0 has exterior capacity 0 . Thus, if $A$ is compact, the subset of $A$ where its equilibrium potential has value $<1$ is a set of exterior capacity 0 . If $A$ is open, and of finite interior capacity, there is a unique 
$\mu$ called the equilibrium distribution, yielding a potential defined by (5.1), called the equilibrium potential, which is identically 1 on $A$. The value $\mu(A)$ is called the capacity of $A$, and is the common value of the interior and exterior capacities. In general, any set in $D_{R}$ with equal finite interior and exterior capacities $c$ will be said to have capacity $c$.

The interior and exterior capacities of a set relative to a circle $C_{R}$ depend on the choice of origin and of $R$, but if a bounded set has zero interior or exterior capacity for one choice of $C_{R}$, it will have this property for every choice, and we shall henceforth omit the phrase "relative to $C_{R}$ " in this connection. If $A$ is an arbitrary plane set, it is said to have interior (or exterior) capacity 0 if every bounded subset has this property.

Theorem 5.1. Let $A$ be a set of zero exterior capacity, and let $z_{0}$ be an arbitrary point. Then the probability that a Brownian path from $z_{0}$ ever meets $A$ for $t>0$ is 0 .

This theorem was proved by Kakutani [11] for $z_{0}$ not in $A$ and $A$ compact. If $z_{0}$ is not in $A, t \geqq 0$ can of course be allowed in the conclusion. It is no restriction to take $A$ bounded, since it is sufficient to prove the theorem for the bounded subsets of $A$. Suppose then that $C_{R}$ is a circle whose interior $D_{R}$ contains the closure of $A$ as well as the point $z_{0}$. By a theorem of Cartan [5], there is a potential $-u$ relative to $C_{R}$, with value $+\infty$ everywhere on $A$, determined by a measure $\mu$ of Borel subsets of some compact subset of $D_{R}$. Then we have seen above that $u$ can be supposed defined and subharmonic not only in $D_{R}$, but in the whole plane, by a suitable extension of its domain of definition, obtaining a function which becomes logarithmically infinite at $\infty$. Since $u$ is upper semicontinuous, it is continuous at every point of $A$, in the obvious extended sense. By Theorem 4.1, if $\{z(t), 0 \leqq t<\infty\}$ is a Brownian motion process starting at $z_{0}$, the process $\{u[z(t)], 0 \leqq t<\infty\}$ is a semimartingale. The proof now proceeds exactly like that of the corollary to Theorem 4.1, which is a special case. Almost all sample functions of the $u[z(t)]$ process are bounded on the set of rational points of every interval $[\delta, 1 / \delta]$ with $\delta>0$. It follows that almost no Brownian path starting from $z_{0}$ can pass through a point of $A$, for $\delta \leqq t \leqq 1 / \delta$. This implies the truth of the theorem.

Theorem 5.2. Let $A$ be a plane set with positive interior capacity, and let $z_{0}$ be an arbitrary point of the plane. Then almost every Brownian path starting at $z_{0}$ meets $A$ for arbitrarily large values of the time parameter.

This theorem is due to Kakutani [11], and its proof is given here only to exhibit the methods of the present paper. In proving it, there is no restriction in assuming that $A$ is compact, and we shall do so. Let $D$ be a disc containing $A$ and $z_{0}$, and let $-u$ be the equilibrium potential of $A$ relative to the boundary of $D$. As in the previous proof, we can suppose that the domain of defini- 
tion of $u$ has been extended to the whole finite plane, on which $u$ is then subharmonic, and continuous on the complement of $A$. Since $u \geqq-1$ in $D$, and since $u$ is upper semicontinuous, $u$ is continuous on $A$, except at the points $z$ with $u(z)>-1$. These points form a set of exterior capacity 0 . Hence almost no Brownian path from $z_{0}$ passes through such a point, and it follows that $u$ is continuous on almost every Brownian path from $z_{0}$. Let $\left\{z^{*}(t), 0 \leqq t<\infty\right\}$ be a Brownian motion process from $z_{0}$, stopped on $A$. According to the corollary to Theorem 4.3 , the process $\left\{u\left[z^{*}(t)\right], 0 \leqq t<\infty\right\}$ is a martingale. The random variables of this martingale are all $\geqq-1$. Hence, according to general martingale theory [7], $\lim _{t \rightarrow \infty} u\left[z^{*}(t)\right]$ exists and is finite with probability 1 . This is obviously impossible unless almost every Brownian path from $z_{0}$ meets $A$. To prove that almost every Brownian path from $z_{0}$ meets $A$ at arbitrarily large values of the parameter, one can proceed as follows. Let $n$ be any positive integer. For each value not in $A$ of the random variable $z(n)$ of the Brownian process not stopped on $A$, the probability is 1 that a Brownian path starting with that value will meet $A$, from what we have just shown. For each value in $A$, this assertion is trivially true. It follows that the probability is 1 that a path from $z_{0}$ will meet $A$ at some time $\geqq n$, and this fact implies the truth of the theorem.

If $0<a<b$, the Brownian paths from some initial point, considered only on the parameter interval $[a, b]$, are almost all continuous arcs, and as such have positive capacity. It follows readily from Theorem 5.2 that then almost every path intersects itself in every parameter interval, in fact that, for almost every path, the part of the path determined by a given parameter interval is intersected by the remainder of the path at arbitrarily large parameter values. This fact was discovered by Lévy [12].

Theorem 5.3. Let $A$ be a subset of an open disc D. Suppose that the capacity of $A$ relative to the boundary $C$ of $D$ is defined, finite, and positive. Then, if $z \in D$ and is not in an exceptional set of exterior capacity 0 , the probability that a Brownian path starting from $z$ meets $A$ for some $t>0$ before it meets $C$ is the value of the equilibrium potential of $A$ relative to $C$, at $z$. If $A$ is compact, or if $A$ is the union of a sequence of compact sets, the exceptional set is empty.

Note that it is not a priori obvious that the probability in question is defined, and that a proof of this fact must be given as part of the proof of the theorem. Suppose first that $A$ is compact, and that $u$ is the equilibrium potential of $A$ relative to $C$. As in the previous proofs, extend the domain of definition of $u$ to make $-u$ subharmonic in the plane, continuous except on a subset of $A$ of zero exterior capacity, and logarithmically infinite at $\infty$. Suppose that $z \in D-A$ and that $\{z(t), 0 \leqq t<\infty\}$ is a Brownian motion process starting from $z$. According to Theorem 4.1 , the process $\{-u[z(t)], 0 \leqq t<\infty\}$ is a semimartingale, and since, by Theorem 5.1, almost no Brownian path meets a discontinuity of $u$, almost all sample functions of the semimartingale 
are continuous. If the semimartingale is stopped when the Brownian path meets $C \cup A$, the resulting process is a martingale on the parameter range $[0, \infty]$, by the corollary to Theorem 4.3 . Hence, if $\left\{z^{*}(t), 0 \leqq t \leqq \infty\right\}$ is the stopped process,

$$
u(z)=E\left\{u\left[z^{*}(\infty)\right]\right\}=P\left\{z^{*}(\infty, \omega) \in A\right\},
$$

so that $u(z)$ is the probability described in the theorem. If $z \in A$ and if $A$ is compact, the quantities in question are both equal to the limit as $n \rightarrow \infty$ of the corresponding quantities for the subset of $A$ at distance $\geqq 1 / n$ from $z$, and are therefore equal. If $A$ has finite capacity relative to $C$, and is the union of a sequence of compact sets, both quantities are equal to the limit of the corresponding quantities for an increasing sequence of compact sets, and are therefore equal. In particular this result can be applied to open subsets of $D$. Now suppose merely that the capacity of $A$ relative to $C$ is defined, finite, and positive. In the following we shall denote by $c(B)$ the capacity of a set $B$ relative to $C$, by $u(B, z)$ the value at $z$ of the equilibrium potential of $B$ relative to $C$. There is a sequence $\left\{B_{n}\right\}$ of compact subsets of $A$ satisfying the relations

$$
B_{1} \subset B_{2} \subset \cdots, \lim _{n \rightarrow \infty} c\left(B_{n}\right)=c(A) .
$$

Then (see Cartan [6] for a justification of the steps of the following argument)

$$
u\left(B_{1}, z\right) \leqq u\left(B_{2}, z\right) \leqq \cdots, \lim _{n \rightarrow \infty} u\left(B_{n}, z\right)=u(A, z) .
$$

There is a sequence $\left\{G_{n}\right\}$ of open sets containing $A$, satisfying the relations

$$
G_{1} \supset G_{2} \supset \cdots, \lim _{n \rightarrow \infty} c\left(G_{n}\right)=c(A) .
$$

Then

$$
u\left(G_{1}, z\right) \geqq u\left(G_{2}, z\right) \geqq \cdots, \lim _{n \rightarrow \infty} u\left(G_{n}, z\right)=u(z) \geqq u(A, z),
$$

and there is equality in the last inequality except possibly on a $z$ set of exterior capacity 0 . If $\Lambda(A, z)$ is the $\Omega$ set corresponding to Brownian paths from $z$ which meet $A$ before $C$,

$$
\Lambda\left(B_{n}, z\right) \subset \Lambda(A, z) \subset \Lambda\left(G_{n}, z\right),
$$

and

$$
P\left\{\Lambda\left(B_{n}, z\right)\right\}=u\left(B_{n}, z\right) \rightarrow u(A, z), \quad P\left\{\Lambda\left(G_{n}, z\right)\right\}=u\left(G_{n}, z\right) \rightarrow u(z) .
$$

Hence $\Lambda(A, z)$ is a measurable $\Omega$ set of measure $u(A, z)$ when $u(z)=u(A, z)$, and this finishes the proof.

Lemma 5.4. Let $D$ be an open disc of radius $r$, with perimeter $C$. Let $A$ be an 
open subset of $D$, with finite capacity $c>0$. If $p(z, A)$ is the probability that a Brownian path starting from $z$ in $D$ meets $A$ before it meets $C$, then

where $\delta$ is the distance from $z$ to $A$.

$$
p(z, A) \leqq c \log \frac{2 r}{\delta},
$$

In fact, if $\mu$ is the equilibrium distribution of $A$ relative to $C$, then

$$
p(z, A)=\int_{A} \log \left|\frac{r^{2}-\bar{z} w}{r(z-w)}\right| \mu(d w) \leqq \mu(A) \log \frac{2 r}{\delta}=c \log \frac{2 r}{\delta} .
$$

TheOREm 5.5. Let $u$ be defined and subharmonic in a plane domain $D$, and let $\boldsymbol{z}$ be an arbitrary point of $D$. Then $u$ is continuous on almost all Brownian paths starting at $z$.

This theorem makes unnecessary part of the hypothesis of Theorem 4.3 and its corollary. Since the set of infinities of $u$ is known to have exterior capacity 0 (see Cartan [5]) the Brownian paths avoid infinities of $u$, according to Theorem 5.1. Since the set of discontinuities of $u$ is not necessarily of zero exterior capacity, the fact that $u$ is continuous on almost all Brownian paths requires a more subtle justification. Suppose that the theorem is known to be true for $D$ the whole plane. Then we shall show that it is true in general. In fact, if $D$ is any domain, it is sufficient to prove that the theorem is true for every subdomain $D_{0}$ of $D$ whose closure is compact and lies in $D$. According to Lemma 2.3 there is a function $u^{*}$ defined on the plane, such that $u^{*}=u$ in $D_{0}$, and that $u^{*}=v+w$, where $w$ is defined and subharmonic in the finite plane, and $v$ is defined on the plane, and continuous except at a finite number of points. Hence, supposing as we are that the theorem is true for $D$ the whole plane, it follows that $w$ is continuous on almost all Brownian paths starting from $z$, which implies the same for $u^{*}$, and therefore also for $u$ while the paths are in $D_{0}$, as was to be proved. Thus we need only prove Theorem 5.5 when $D$ is the plane, and we shall make this assumption from now on. Let $\{z(t)$, $0 \leqq t<\infty\}$ be a Brownian motion process starting from $z$. In the following, the open disc with center $z$ and radius $r$ will be denoted by $D_{r}$, its perimeter by $C_{r}$. According to a theorem of Cartan [5], if $0<r_{1}<r_{2}<r$, there is an open subset $A$ of $D_{r_{2}}-D_{r_{1}}$, of arbitrarily small capacity relative to $C_{r}$, with the property that, if $B=D_{r_{2}}-A$, then $u$ is continuous on $B$ relative to $B$. By Lemma 5.4, the probability that a Brownian path from $z$ meets $C_{r}$ before it meets $A$ is nearly 1 if the capacity of $A$ is small relative to $C_{r}$. Hence the probability that a Brownian path from $z_{0}$ lies entirely in $B$, and therefore yields a continuous sample function of the $u[z(t)]$ process between the first time the path meets $C_{r_{2}}$ and the last time before that when the trajectory meets $C_{r_{1}}$, can be made arbitrarily near 1 by a proper choice of $B$. It follows that almost all sample functions of the $u[z(t)]$ process are continuous in the 
stated interval. Since $r_{1}$ can be made arbitrarily small and $r_{2}$ arbitrarily large, and since we have already seen that almost no Brownian path from $z$ ever returns to $z$, we have now proved that $u$ is continuous on almost every Brownian path from $z$, except possibly at $t=0$. In discussing continuity at $t=0$, we need only discuss the case when $u\left(z_{0}\right)$ is finite. (Otherwise $u$ is continuous at $z_{0}$ in the obvious extended sense, and there is the same kind of continuity on almost every Brownian path at $t=0$.) We can also assume that $u$ satisfies the condition (4.1) of Theorem 4.1, because if it does not, we can, with the help of Lemma 2.2, change $u$ outside a disc containing $z$ in such a way that this condition will be satisfied. Assuming, then, that (4.1) is satisfied, the process $\{u[z(t)], 0 \leqq t<\infty\}$ is a semimartingale, and we have already proved that almost all sample functions of this semimartingale are continuous for $t>0$. Now since $u$ is subharmonic,

$$
\limsup _{\zeta \rightarrow z} u(\zeta)=u(z) .
$$

On the other hand, according to the general theory of semimartingales [7], $\lim _{t \rightarrow 0} u[z(t)]$ exists and is finite with probability 1 . Hence

$$
\lim _{t \rightarrow 0} u[z(t)] \leqq u(z)
$$

with probability 1 , whereas it is known, again from the general theory of semimartingales, that

$$
E\left\{\lim _{t \rightarrow 0} u[z(t)]\right\} \geqq E\{u[z(0)]\}=u(z) .
$$

Combining these two inequalities, we find that

$$
\lim _{t \rightarrow 0} u[z(t)]=u(z)
$$

with probability 1 , as was to be proved.

Cartan [6] has introduced a topology in the plane, the "fine" topology, the coarsest topology making all subharmonic functions continuous. In the following, when a topological concept is used without specification of the topology, the ordinary plane topology will be meant. Cartan has shown that, to every fine neighborhood of a point $z_{0}$ there correspond a neighborhood of $z_{0}$, a function $u$ defined and subharmonic in this neighborhood, finite at $z_{0}$, and a positive $\delta$, such that the fine neighborhood includes all points of the ordinary neighborhood which satisfy the inequality

$$
u(z)>u\left(z_{0}\right)-\delta .
$$

Conversely, every $z$ set satisfying such an inequality intersects a sufficiently small neighborhood of $z_{0}$ in a fine neighborhood of $z_{0}$. (Actually Cartan uses potentials rather than subharmonic functions in his discussion, obtaining a trivially equivalent characterization of a fine neighborhood.) 
Brelot [2] introduced the concept of thinness of a set at a point. In terms of the fine topology, a point set is thin at a point $z$ if $z$ is an isolated point (fine topology) of the set $A \cup\{z\}$. For example, if a point set has zero exterior capacity it is thin at every point of the plane. We shall use below the fact that if $A$ is compact, or more generally if $A$ is the union of a sequence of compact sets, and if $A$ is not thin at a point $z$, then the equilibrium potential (or inner equilibrium potential if the capacity of $A$ is undefined) of $A$ relative to any containing circle is 1 at $z$. This is a familiar fact if $A$ is compact. In the general case the potential in question is the limit of a monotone nondecreasing sequence of potentials, the equilibrium potentials of a corresponding sequence of compact subsets of $A$. The latter potentials are 1 on their sets, excluding at most a set of exterior capacity 0 . Then $u$ is one on $A$ except possibly for such an exceptional set. There is a fine neighborhood of $z$ so small that it contains no point of this exceptional set (except perhaps $z$ itself), since the exceptional set is thin at $z$. On the other hand every fine neighborhood of $z$ contains a point of $A$ other than $z$, by hypothesis. Hence every fine neighborhood of $z$ contains a point where $u$ has the value 1 . Since $u$ is continuous in the fine topology, it follows that $u(z)=1$.

TheOREM 5.6. If a set $A$ is thin at a point, almost no Brownian path from that point meets $A$ for small positive parameter values.

If $A$ is thin at $z_{0}$, there is a subharmonic function defined in a neighborhood of $z_{0}$, and positive numbers $\delta_{1}, \delta_{2}$, such that the inequalities

$$
u(z)>u\left(z_{0}\right)-\delta_{1}, \quad\left|z-z_{0}\right|<\delta_{2}
$$

define a fine neighborhood of $z_{0}$ containing no point of $A$ except possibly $z_{0}$. According to Theorem 5.5, for almost all $\omega, u[z(t, \omega)]$ defines a continuous function of $t$ for small parameter values, for example until the Brownian paths reach distance $\delta_{2}$ from $z_{0}$. Hence, for almost all $\omega$, the inequalities

$$
u[z(t, \omega)]>u\left(z_{0}\right)-\delta_{1}, \quad\left|z(t, \omega)-z_{0}\right|<\delta_{2}
$$

hold for sufficiently small $t$, depending on $\omega$. That is, almost every Brownian path from $z_{0}$ remains in the specified fine neighborhood of $z_{0}$ for sufficiently small $t$, and thus, neglecting $z_{0}$ itself, does not meet $A$ in this fine neighborhood, as was to be proved. The following theorem provides a partial converse to this result.

THEOREM 5.7. Let $A$ be a compact set or the union of a sequence of compact sets, and let $p(z)$ be the probability that a Brownian path from $z$ meets $A$ for arbitrarily small positive values of $t$. Then if $A$ is thin at $z, p(z)=0$; if $A$ is not thin at $z, p(z)=1$.

The fact that $p(z)$ can only have the values 0,1 can be deduced from the zero-one law of the theory of probability, but we shall not use this approach. 
Before proving the theorem, we give two examples. If $z$ is an isolated point of $A$, almost no Brownian path reaches $A$ for small positive parameter values. Thus $p(z)=0$ in this case, and $A$ is thin at $z$, as is of course obvious from the definition. As a second example, let $B$, the complement of $A$, be an open disc less a radius, and let $z$ be the center. Then almost every Brownian path from $z$ meets both $B$ and the radius at arbitrarily small times. Hence $p(z)=1$, and $A$ is not thin at $z$. In view of the previous theorem, it is sufficient to prove that if $A$ is not thin at $z$ then $p(z)=1$. If $p(z)<1$, there is a circle $C$ with center $z$, so small that the probability that a Brownian path from $z$ meets $A$ before $C$ is less than 1 . Then by Theorem 5.2 the inner equilibrium potential of the part of $A$ in $C$ relative to $C$ is less than 1, and we have remarked above that this is impossible unless $A$ is thin at $z$.

The concept of regularity for the Dirichlet problem has an elegant form in probability language. Let $B$ be a bounded domain, with complement $A$. Let $f$ be a function defined and continuous on the boundary of $B$. There is then a "Dirichlet solution," a function defined and harmonic in $B$, with limiting boundary function $f$, except possibly at a set of boundary points of exterior capacity 0 , the irregular points. These are the points where $A$ is thin. (See Brelot [2].) Thus we can say that a finite point $z$ is irregular for the Dirichlet problem if and only if $\left(^{1}\right)$ almost every Brownian path from $z$ lies in the domain $B$ for an interval of time before it meets $A$ (not counting the initial position).

6. Application to boundary value problems. Let $u$ be a function defined and subharmonic in the unit disc $D:|z|<1$, and suppose that

$$
\sup _{r<1} \int_{0}^{2 \pi}\left|u\left(r e^{i \theta}\right)\right| d \theta<\infty .
$$

Then by a theorem of Littlewood [14], $u$ has finite boundary values on approach to the bounding perimeter along almost all radii, that is, $\lim _{r \rightarrow 1} u\left(r e^{i \theta}\right)$ exists and is finite for almost all $\theta$ (Lebesgue measure). To prove this result, it is shown first that, under the hypothesis on $u, u$ can be written as the sum of a subharmonic function $u_{1}$ which is the potential of a negative mass distribution relative to the perimeter of $D$

$$
u_{1}(z)=-\int_{D} \log \left|\frac{1-z \bar{w}}{z-w}\right| \mu(d w)
$$

and a harmonic function $u_{2}$ which satisfies (6.1). Here $\mu$ is a measure of Borel subsets of $D$, finite on every compact set, and $u_{1}$ satisfies (6.1), or, equivalently,

${ }^{1}$ A. Dvoretzky has apparently also derived this characterization of regular and irregular points. [See Kac, Proceedings of the Second Berkeley Symposium on Mathematical Statistics and Probability, 1951, p. 211.] 


$$
\int_{D}(1-|w|) \mu(d w)<\infty .
$$

We note for later use that it is then easily concluded that

$$
\lim _{r \rightarrow 1} \int_{0}^{2 \pi} u_{1}\left(r e^{i \theta}\right) d \theta=0 .
$$

Since $u_{1} \leqq 0$, this means that $u_{1}\left(r e^{i \theta}\right) \rightarrow 0$ in measure when $r \rightarrow 1$. It was shown by Littlewood that actually $\lim _{r \rightarrow 1} u_{1}\left(r e^{i \theta}\right)=0$ for almost all $\theta$. Counterexamples show that there need not be a limit of $u_{1}$ on approach to the perimeter along nontangential paths. However Tolsted [17] has shown that if $L$ is a continuous curve, in $|z|<1$ except for one end point on the perimeter, at which $L$ is tangent to a chord of the perimeter, and if $L_{\theta}$ is $L$ rotated about the origin through an angle $\theta$, then $u$ has a finite limit when $z$ approaches the perimeter on $L_{\theta}$ for almost all $\theta$. The boundary values for $u_{2}$ are obtained more simply, since according to a much older theorem of $F$. Riesz there are unique boundary values of $u_{2}$ at almost every perimeter point in terms of arbitrary nontangential approach.

Littlewood's theorem, and Tolsted's generalization, give the existence of boundary values on approach to the perimeter along certain classes of curves. There are obvious difficulties inherent in such theorems if they are to be extended to domains with irregular bounding curves. We shall prove a theorem of this same general nature which is just as easy to prove for an arbitrary domain as for a disc. The fact seems to be that Brownian paths are adapted to subharmonic functions. The continuity of any subharmonic function on almost all Brownian paths is one indication of this fact. Theorem 6.1, which states roughly that Brownian paths to the boundary of a domain almost always approach the boundary in just the right way to make limiting values of a subharmonic function possible, is another indication of this fact.

Let $D$ be any plane domain, and let $u$ be defined and subharmonic in $D$. Then $u$ is said to have a harmonic majorant in $D$ if there is a function $v$, defined and harmonic in $D$, such that $u \leqq v$. If $D$ is the unit disc, (6.1) is known to be necessary and sufficient for the existence of a positive harmonic majorant. We shall discuss the existence of boundary values for subharmonic functions with positive harmonic majorants. We observe that if the complement of the domain in question has exterior capacity 0 , a function defined, harmonic, and positive in the domain can be extended to be defined, harmonic, and positive on the entire plane, and is therefore identically a positive constant. A subharmonic function defined in this domain, with a positive harmonic majorant, is therefore bounded from above by a positive constant. It too can then be extended to be defined on the whole plane, and must be identically a constant. Thus only a trivial special case is excluded in the following theorem. 
TheOREM 6.1. Let $D$ be any plane domain whose complement has positive exterior capacity, and let $u$ be a function defined and subharmonic in $D$, with a positive harmonic majorant. Then, if $z_{0} \in D, u$ has a finite limit along almost every Brownian path to the boundary, starting from $z_{0}$.

We note that, by our hypothesis on $D$, which implies that its boundary is of positive interior capacity, almost every Brownian path from $z_{0}$ actually meets the boundary. Let $v$ be a positive harmonic majorant of $u$. Let $D_{n}$ be, for each positive integer $n$, a bounded domain, and suppose that.

$$
D_{1} \subset D_{2} \subset \cdots, \bigcup_{1}^{\infty} D_{n}=D_{\infty}=D \text {, }
$$

and that the closure of $D_{n}$ lies in $D$. Let $\left\{z_{n}(t), 0 \leqq t \leqq \infty\right\}$, for $n \leqq \infty$, be the Brownian motion process starting at $z_{0}$, and stopped on the boundary of $D_{n}$. These processes are to be successive stopped versions of one unstopped process. Then if $n<\infty$, the processes

$$
\left\{u\left[z_{n}(t)\right], 0 \leqq t<\infty\right\}, \quad\left\{v\left[z_{n}(t)\right], 0 \leqq t \leqq \infty\right\}
$$

are respectively a semimartingale and martingale, by Theorem 4.3 , if we suppose, as we shall for the present, that $u\left(z_{0}\right)>-\infty$. Defining $K=2 v\left(z_{0}\right)-u\left(z_{0}\right)$, it follows that

$$
\begin{aligned}
E\left\{\left|u\left[z_{n}(t)\right]\right|\right\} & \leqq E\left\{v\left[z_{n}(t)\right]-u\left[z_{n}(t)\right]\right\}+E\left\{v\left[z_{n}(t)\right]\right\} \\
& \leqq 2 v\left(z_{0}\right)-u\left(z_{0}\right)=K .
\end{aligned}
$$

If $t_{1}<\cdots<t_{j}$, and if $r_{1}<r_{2}$, the number of times the succession

$$
u\left[z_{n}\left(t_{1}\right)\right], \cdots, u\left[z_{n}\left(t_{j}\right)\right]
$$

goes from below $r_{1}$ to above $r_{2}$ is a random variable, and according to a theorem of Snell [16] this random variable has expectation at most

$$
\frac{E\left\{\left|u\left[z_{n}\left(t_{j}\right)\right]\right|\right\}+\left|r_{1}\right|}{r_{2}-r_{1}} \leqq \frac{K+\left|r_{1}\right|}{r_{2}-r_{1}} \quad(n<\infty) .
$$

Let $\Lambda_{n}$ be the $\Omega$ set corresponding to continuous sample functions of the $u\left[z_{n}(t)\right]$ process for which the number of times the sample function proceeds from below $r_{1}$ to above $r_{2}$ before the Brownian path meets the boundary of $D_{n}$ is at least $q$. Then it is clear that, without going into the question of the measurability of $\Lambda_{n}$, it has outer measure (lower limit of the measures of measurable containing sets) at most

$$
\frac{K+\left|r_{1}\right|}{q\left(r_{2}-r_{1}\right)}
$$

Then the outer measure of $\bigcup_{n} \Lambda_{n}$ is at most this same number, so that, if $\tau$ is 
the first value of $t$ at which a Brownian path from $z_{0}$ meets the boundary of $D$, almost no $u\left[z_{\infty}(t)\right]$ process sample function has infinitely many crossings from below $r_{1}$ to above $r_{2}$, for $t<\tau$. Letting $r_{1}, r_{2}$ run through all rational values, with $r_{1}<r_{2}$, we find that almost no sample function of the $u\left[z_{\infty}(t)\right]$ process has an oscillatory character as $t \rightarrow \tau$, that is,

$$
\lim _{t \rightarrow r(\omega)} u\left[z_{\infty}(t, \omega)\right]=y(\omega)
$$

exists with probability 1 . Since

$$
E\left\{\left|u\left[z_{n}(\infty)\right]\right|\right\} \leqq K,
$$

and since

$$
\lim _{n \rightarrow \infty} u\left[z_{n}(\infty)\right]=y
$$

with probability 1 , it follows that $E\{|y|\} \leqq K$. Hence $y$ is finite with probability 1 . Finally, consider the case when $u(z)=-\infty$. In this case, we replace $u$ in a small disc, with center $z$, by a function harmonic in the disc, the solution of the Dirichlet problem in the disc, with boundary function $u$ on the perimeter. The application of the result already proved to this modified function, which is subharmonic and has the same positive harmonic majorant as $u$, yields the stated result for the given function.

The proof of Theorem 6.1 is now complete. To tie it in with Littlewood's result, we remark that, if $D$ is the unit disc, and if $u_{1}$ is given by (6.2), where $\mu$ is a measure as described, so that $u_{1}$ satisfies (6.1), then the boundary value is 0 along almost every Brownian path. To prove this, let $D_{n}$ as used above be the disc $|z|<1-1 / n$, and suppose that $z_{0}=0$. Then, using (6.4) and the obvious fact that $z_{n}(\infty)$ is distributed uniformly over the perimeter $|z|=1-1 / n$, we find that

$$
E\left\{u_{1}\left[z_{n}(\infty)\right]\right\}=\frac{1}{2 \pi} \int_{0}^{2 \pi} u_{1}\left[(1-1 / n) e^{i \theta}\right] d \theta \rightarrow 0, \quad n \rightarrow \infty .
$$

Since $u_{1} \leqq 0$, it follows that $u_{1}\left[z_{n}(\infty)\right] \rightarrow 0$ in measure, so that its probability 1 limit, the boundary function $y$ obtained above, must also be 0 . If $z_{0} \neq 0$, the argument is very slightly more complicated, because $z_{n}(\infty)$ has a Poisson kernel density function on the circle $|z|=1-1 / n$, and we omit the details.

Let $D$ be any plane domain whose complement is of positive exterior capacity, and let $C$ be the boundary of $D$. Then, for a large class of functions $f$ defined on $C$, there is a unique solution to the Dirichlet problem of finding a function harmonic in $D$ with the boundary function $f$ on $C$. More specifically, following Brelot $[1 ; 3]$, there is, corresponding to each point $z$ of $D$, a measure $\mu(z, \cdot)$ of Borel subsets of $C$, with $\mu(z, C)=1$, called the harmonic measure of boundary sets relative to $z$, and the Dirichlet problem solution is determined by harmonic measure in the following way. (We shall suppose 
below that the harmonic measure relative to $z$ has been completed, so that it is defined on a slightly larger class than the class of Borel subsets of $C$. This class is known to be independent of $z$, and the class of functions measurable and absolutely integrable with respect to harmonic measure is also independent of $z$. The functions of this class will be called admissible boundary functions below.) To each admissible boundary function corresponds a unique harmonic function, according to Brelot's treatment, the solution of the generalized Dirichlet problem, defined by

$$
u(z)=\int_{C} f(w) \mu(z, d w) .
$$

One of the problems of this theory is to establish a simple and elegant sense in which an admissible boundary function is really the boundary function of the Dirichlet solution, aside from the sense derived from Brelot's treatment. For example, if the boundary function $f$ is continuous at a point, and if that point is regular, then the Dirichlet solution has a boundary value at that point in the usual sense, the value of $f$ there. Moreover the set of irregular points has zero exterior capacity. Since all bounded Baire functions are admissible, it is clear that no result as simple as this can be true for all admissible boundary functions, but the probability analysis we shall give exhibits a connection nearly as simple. To clarify the significance of this analysis, we shall make a few remarks on the case when $D$ is the unit disc. In this case, the harmonic measure of a perimeter set $A$ relative to $z$ is given by

$$
\mu(z, A)=\frac{1}{2 \pi} \int_{A} \frac{1-r^{2}}{1-2 r \cos (\theta-\phi)+r^{2}} d \phi, \quad z=r e^{i \theta} .
$$

The admissible boundary functions are the functions which are Lebesgue measurable and integrable on the perimeter, and, if $f$ is admissible, the corresponding harmonic function $u$ is then given by the Poisson integral,

$$
u\left(r e^{i \theta}\right)=\frac{1}{2 \pi} \int_{0}^{2 \pi} f\left(e^{i \phi}\right) \frac{1-r^{2}}{1-2 r \cos (\theta-\phi)+r^{2}} d \phi .
$$

It is known that in this case $u$ has the boundary function $f$ in the sense of approach to the boundary along (almost all) radii, and in fact any nontangential approach is legitimate in this case. It is natural to expect a corresponding result for more complicated domains with a suitable generalization of nontangential approach. It is interesting that approach to the boundary along Brownian paths will give a corresponding result for an arbitrary domain, according to the following theorem.

THEOREM 6.2. Let $D$ be any plane domain of the extended plane whose complement has positive exterior capacity, let $f$ be an admissible boundary function, and let $u$ be the corresponding Dirichlet problem solution. Let $z_{0} \neq \infty$ be a point 
of $D$, and let $\left\{z_{\infty}(t), 0 \leqq t \leqq \infty\right\}$ be the Brownian motion process starting from $z_{0}$ and stopped on $C$. Then $u$ has the boundary limit $f$ on almost every Brownian path to the boundary from $z$, and, defining $u(z)=f(z)$ on $C$, the process

$$
\left\{u\left[z_{\infty}(t)\right], 0 \leqq t \leqq \infty\right\}
$$

is a martingale, so that

$$
E\left\{u\left[z_{\infty}(\infty)\right]\right\}=E\left\{f\left[z_{\infty}(\infty)\right]\right\}=u(z) .
$$

According to this theorem, the Brownian motion solves the Dirichlet problem whenever this problem has a solution, and gives the simplest possible explicit relation between solution and boundary function. The random variable $z_{\infty}(\infty)$ determines a distribution on $C$, and $u(z)$ becomes the weighted average of the boundary function in accordance with this distribution. The distribution of $z_{\infty}(\infty)$ is thus the harmonic measure relative to $z$, a fact due to Kakutani [11] for special domains. The fact that the values of the boundary function at the irregular points of the boundary are irrelevant corresponds to the fact that almost no Brownian path meets the boundary in an irregular point (because the set of irregular points has zero exterior capacity). We can, and shall, assume in the following that the point $\infty$ is not in $D$, removing it from $D$ if it is initially there.

There are two ways available at this stage to prove this theorem. One can define $u(z)$ by (6.7) (ignoring the first term), and prove that as so defined $u$ has the stated properties, or one can define $u$ as the Dirichlet solution and prove (6.7) and the stated relation of $u$ to $f$. We shall use the second method. Suppose then that $f$ is admissible and that $u$ is the corresponding Dirichlet solution. It follows that $u$ has a positive harmonic majorant, the Dirichlet solution corresponding to the boundary function $|f|$. Theorem 6.1 is now applicable, and asserts that $u$ has a boundary value along almost every Brownian path from $z$ to the boundary. There remains the proof that the boundary function defined in this way is the given boundary function $f$, and that (6.7) is true. In particular, if $f$ is continuous, $u$ has the boundary value $f$ on the set of regular boundary points, so that the boundary function along Brownian paths is $f$, with probability 1 . Hence, using the notation of the proof of Theorem 6.1,

$$
\lim _{n \rightarrow \infty} u\left[z_{n}(t)\right]=u\left[z_{\infty}(t)\right]
$$

with probability 1 . According to Theorem 4.3 , the process $\left\{u\left[z_{n}(t)\right], 0 \leqq t \leqq \infty\right\}$ is a martingale relative to a certain family of Borel fields $\left\{G_{n}(t), 0 \leqq t \leqq \infty\right\}$, a family determined by the Brownian motion process and $D_{n}$ but not dependent on $u$, that is not dependent on $f$. Moreover the $u\left[z_{n}(t)\right]$ process can be considered as obtained from the $u\left[z_{n+1}(t)\right]$ process by stopping on the boundary of $D_{n}$. Hence, as is easily verified by an examination of the stopping 
procedure (see [7]), $G_{n}(t) \subset G_{n+1}(t)$. The fact that the $u\left[z_{n}(t)\right]$ process is a martingale can be expressed by the equality

$$
\int_{\Lambda} u\left[z_{n}(t)\right] d P=\int_{\Lambda} u\left[z_{n}(\infty)\right] d P, \quad \Lambda \in G_{n}(t) .
$$

Now $u$ is bounded, since $f$ is continuous on $C$, considered as a point set in the extended plane. Hence, fixing $\Lambda \in \cup_{m} G_{m}(t)$, and letting $n \rightarrow \infty$ in this equality, we find that

$$
\int_{\Lambda} u\left[z_{\infty}(t)\right] d P=\int_{\Lambda} u\left[z_{\infty}(\infty)\right] d P .
$$

It follows, if $G_{\infty}(t)$ is the Borel field generated by $\cup_{m} G_{m}(t)$, that this equality is true for all $\Lambda \in G_{\infty}(t)$, and this means that the $u\left[z_{\infty}(t)\right]$ process is a martingale relative to the family $\left\{G_{\infty}(t), 0 \leqq t \leqq \infty\right\}$. We write this fact in the form

$$
E\left\{f\left[z_{\infty}(\infty)\right] \| G_{\infty}(t)\right\}=u\left[z_{\infty}(t)\right]
$$

true, with probability 1 , for each $t \geqq 0$. This discussion has been for $f$ continuous. Now consider the class $H$ of admissible boundary functions, and the subclass $H_{1}$ for which (6.8) is true with probability 1 for each non-negative $t$. We shall prove that $H=H_{1}$. We have shown that $H_{1}$ includes the continuous admissible functions. Now any subset $H_{0}$ of $H$ is $H$ itself if $H_{0}$ satisfies the following four conditions.

1. $H_{0}$ includes the continuous functions.

2. $H_{0}$ is a linear class.

3. If $\left\{f_{n}\right\}$ is a monotone nondecreasing sequence of non-negative functions in $H_{0}$, and if $\left\{u_{n}\right\}$ is the corresponding sequence of Dirichlet solutions, then the limit $f$ of $\left\{f_{n}\right\}$ is in $H_{0}$ unless $\lim _{n \rightarrow \infty} u_{n}=+\infty$ in $D$.

4. If $f \leqq g \leqq h$, if $f$ and $h$ are in $H_{0}$, and if $f$ and $h$ correspond to the same Dirichlet solution, then $g$ is also in $H_{0}$.

To prove that $H_{1}=H$, we prove that $H_{1}$ satisfies these four conditions. We have already verified that the first is satisfied. If $f_{1}, f_{2} \in H_{1}$, and if $c_{1}, c_{2}$ are constants,

$$
E\left\{f_{i}\left[z_{\infty}(\infty)\right] \| G_{\infty}(t)\right\}=u_{i}\left[z_{\infty}(t)\right]
$$

with probability 1 , where $u_{i}$ is the Dirichlet solution corresponding to $f_{i}$. Then $c_{1} f_{1}+c_{2} f_{2}$ has the Dirichlet solution $c_{1} u_{1}+c_{2} u_{2}$, and

$$
\begin{aligned}
& E\left\{\left(c_{1} f_{1}+c_{2} f_{2}\right)\left[z_{\infty}(\infty)\right] \| G_{\infty}(t)\right\} \\
& =c_{1} E\left\{f_{1}\left[z_{\infty}(\infty)\right] \| G_{\infty}(t)\right\}+c_{2} E\left\{f_{2}\left[z_{\infty}(\infty)\right] \| G_{\infty}(t)\right\} \\
& =c_{1} u_{1}\left[z_{\infty}(t)\right]+c_{2} u_{2}\left[z_{\infty}(t)\right]
\end{aligned}
$$

with probability 1 , so that $c_{1} f_{1}+c_{2} f_{2} \in H_{1}$, and $H_{1}$ thus satisfies the second condition. Under the hypotheses of the third condition, the sequence $\left\{u_{n}\right\}$ is 
monotone in $D$. Let $u$ be its limit, and suppose that $u$ is not identically $+\infty$. Then

$$
E\left\{f_{n}\left[z_{\infty}(\infty)\right] \| G_{\infty}(t)\right\}=u_{n}\left[z_{\infty}(t)\right],
$$

with probability 1 . Now $\left\{f_{n}\left[z_{\infty}(\infty)\right], n \geqq 1\right\}$ is a monotone sequence of random variables, which are non-negative, and have expectations given by

$$
E\left\{f_{n}\left[z_{\infty}(\infty)\right]\right\}=E\left\{u_{n}\left[z_{\infty}(t)\right]\right\}=u_{n}(z),
$$

using the martingale property of the $u_{n}\left[z_{\infty}(t)\right]$ process. The sequence $\left\{u_{n}\right\}$ is a monotone sequence of non-negative harmonic functions. The limit $u$ is therefore identically $+\infty$ or a harmonic function. Under the present hyhypotheses, $u$ must be harmonic, so $E\left\{f_{n}\left[z_{\infty}(\infty)\right]\right\}$ defines a bounded sequence. Hence, according to the known properties of conditional expectations [7],

$$
\lim _{n \rightarrow \infty} E\left\{f_{n}\left[z_{\infty}(t)\right] \| G_{\infty}(t)\right\}=E\left\{f\left[z_{\infty}(\infty)\right] \| G_{\infty}(t)\right\}
$$

with probability 1 . If we define $u(z)=f(z)$ on $C$, then $u_{n} \rightarrow u$ on $D \cup C$, so that $u_{n}\left[z_{\infty}(t)\right] \rightarrow u\left[z_{\infty}(t)\right]$. Thus (6.9) becomes, when $n \rightarrow \infty$, the desired relation (6.8), and we have proved that $H_{1}$ satisfies the third condition. The fourth condition is obviously satisfied by $H_{1}$. Hence $H_{1}=H$, and we have proved that the $u\left[z_{\infty}(t)\right]$ process is a martingale on the parameter interval $[0, \infty]$. We have not yet proved, however, that the sample functions of the process are continuous, so that we do not know that $u$ has the boundary function $f$ as a limit along Brownian paths to $C$. Let $H_{2}$ be the subclass of $H$ for which this is true. We know that $H_{2}$ satisfies the first condition above, that is, that the continuous boundary functions are in $\mathrm{H}_{2}$, and the second and fourth conditions are also obviously satisfied. To prove that $H_{2}$ satisfies the third condition we use the same notation as in our previous discussion of this condition. If $m<n$, the sample functions of the $u_{n}\left[z_{\infty}(t)\right]-u_{m}\left[z_{\infty}(t)\right]$ process are almost all continuous. Hence, according to a standard martingale inequality, if $\lambda>0$,

$$
\begin{aligned}
P\left\{\sup _{0 \leqq t \leqq \infty}\left(u_{n}\left[z_{\infty}(t)\right]-u_{m}\left[z_{\infty}(t)\right]\right) \geqq \lambda\right\} & \leqq \frac{1}{\lambda} E\left\{u_{n}\left[z_{\infty}(\infty)\right]-u_{m}\left[z_{\infty}(\infty)\right]\right\} \\
& =\frac{1}{\lambda}\left[u_{n}\left(z_{0}\right)-u_{m}\left(z_{0}\right)\right] .
\end{aligned}
$$

It follows that the sample functions of the $u_{n}\left[z_{\infty}(t)\right]$ process converge uniformly to those of the $u\left[z_{\infty}(t)\right]$ process, with probability 1 , and that therefore almost all sample functions of the latter process are continuous, as was to be proved.

Kakutani [11] has considered the following problem. Let $D$ be a domain of the extended plane, with boundary $C$, and let $A$ be a subset of $C$. If $z \in D$, the problem is to find the probability that a Brownian path from $z$ meets $A$ 
before it meets $C-A$. It is supposed that $C$ has positive exterior capacity, in order that a Brownian path from $z$ will be sure to meet $C$. Define the function $f$ on $C$ to be equal to 1 on $A$ and 0 otherwise. Then if $f$ is an admissible boundary function in the sense used above, if $u$ is the corresponding Dirichlet solution, and if $\left\{z_{\infty}(t), 0 \leqq t \leqq \infty\right\}$ is a Brownian motion process from $z$, stopped on $C$, we have seen that $u(z)$ is the harmonic measure of $A$ relative to $z$, and that

$$
u(z)=E\left\{u\left[z_{\infty}(\infty)\right]\right\}=P\left\{z_{\infty}(\infty, \omega) \in A\right\} .
$$

The last term in this continued equality is the desired probability. This reasoning is valid if and only if $f$ is an admissible boundary function, that is, if and only if $A$ is measurable in terms of harmonic measure. Kakutani announced this result under somewhat more stringent conditions on $C$ and $A$.

Let $D$ be an open disc less a radius. Then one can consider the points of the radius other than the center as double boundary points of $D$, differentiating between the two boundary points with the same coordinate by means of the two different possible approaches, from one side or the other. A generalized Dirichlet problem is easily formulated in which the boundary function may have different values on the different "sides" of the radius. More generally Brelot [4] has done this for general domains, and Theorem 6.2 and its proof go over with no change to cover this problem, called the "ramified" Dirichlet problem.

The following theorem can be proved for more general domains, but the generalization will not be necessary for the purposes of this paper.

Theorem 6.3. Let $D$ be a bounded plane domain, and let $\left\{D_{s}, s \in S\right\}$ be a family of subdomains, with $D_{s_{1}} \subset D_{s_{2}}$ for $s_{1}<s_{2}$, whose closures lie in $D$. Let $z$ be a point in every $D_{s}$, and let w(s) be the first point of $D$ at which a Brownian path from z meets the boundary of $D_{s}$. Let $u$ be defined and subharmonic [har. monic or regular] in D. If $u$ is subharmonic, it is also supposed that, for each $s$, $u$ is integrable on the boundary of $D_{s}$ with respect to the harmonic measure on this boundary relative to $z$. Then the process $\{u[w(s)], s \in S\}$ is a semimartingale [martingale]. If $u(z)>-\infty$, the theorem remains true with a new parameter value adjoined to $S$, to the left of all its other elements, defining $w=z$ at this value.

As usual, it will be sufficient to prove the theorem for $u$ subharmonic, and we shall restrict our attention to this case. Suppose first that $u$ is bounded on every compact subset of $D$. This condition will of course be satisfied if $u$ is continuous. Let $s_{0}$ be a point of $S$. It will be sufficient to prove that the $u[w(s)]$ process with $s \leqq s_{0}$ is a semimartingale. Let $\left\{z^{*}(t), 0 \leqq t \leqq \infty\right\}$ be a Brownian motion process starting from $z$, stopped on the boundary of $D_{s_{0}}$. According to Theorem 4.3 , the process $\left\{u\left[z^{*}(t)\right], 0 \leqq t \leqq \infty\right\}$ is a semimartingale. The $u[w(s)]$ process for $s \leqq s_{0}$ is obtained from this process by optional sampling (see [7] for a discussion of this operation) and hence is also a semi- 
martingale, as was to be proved. In fact the requirements for this invariance of the semimartingale property stated in the reference are obviously satisfied in the present case. If $u$ is not necessarily bounded on every compact subset of $D$, it is nevertheless bounded from above on every such set, because $u$ is subharmonic. Hence, if $u$ is replaced by $u_{n}=\max (u, n), u_{n}$ is subharmonic in $D$, and bounded on every compact subset of $D$. Hence, according to what we have just proved, the process $\left\{u_{n}[w(s)], s \in S\right\}$ is a semimartingale. Since $u_{n}[w(s)]$ converges monotonely to $u[w(s)]$ when $n \rightarrow \infty$, we find that the $u[w(s)]$ process is also a semimartingale if $E\{|u[w(s)]|\}$ is finite on $S$. The hypothesis that $u$ be integrable with respect to the harmonic measure relative to $z$ on the boundary of each $D_{s}$ is precisely this hypothesis of a finite expectation in different language. Finally, if $u(z)<\infty$, the stopping at the boundary of each $D_{s}$, which led to $u[w(s)]$, can be augmented by stopping at $z$ itself, leading to a random variable identically equal to $u(z)$ as the first random variable of the augmented semimartingale.

As an application of Theorem 6.3 we shall discuss Poisson kernel processes and their generalizations. By a Poisson kernel process we mean any stochastic process with parameter interval $[0, \infty)$, whose random variables are taken modulo $2 \pi$, and whose probability relations are determined as follows. The process is to be a Markov process; there is no restriction on the initial distribution, that of $x(0)$; the conditional distribution of $x(t)$ given that $x(s, \omega)$ $=\xi, s<t$, is determined by the density (in the variable $\eta$ )

$$
K(s, t, \eta-\xi)=\frac{1}{2 \pi} \frac{t^{2}-s^{2}}{t^{2}-2 s t \cos (\eta-\xi)+s^{2}} .
$$

Poisson kernel processes were apparently first discussed by Hostinsky [9]. Since the transition density is, for fixed $s, t$, a function of $\eta-\xi$, the $x(t)$ process will have independent increments. If $x(0)$ is distributed uniformly on $[0,2 \pi), x(t)$ will have this same distribution. If $y(t)=x\left(e^{t}\right)$, the $y(t)$ process, with parameter interval $(-\infty, \infty)$, is also a Markov process with independent increments, and if $x(0)$ is uniformly distributed on $[0,2 \pi)$, the corresponding $y(t)$ process is stationary.

Lévy [12] has investigated the continuity properties of processes with independent increments whose random variables have values modulo $2 \pi$. Under the usual separability assumptions, almost all the sample functions of such a process, if properly centered, are continuous except for nonoscillatory discontinuities (jumps). Moreover the distribution of any increment $x(t)$ $-x(s)$ of such a process, which in the Poisson kernel case is given by the density $K(s, t, \cdot)$, is obtained by reducing an infinitely divisible distribution modulo $2 \pi$. Finally, almost all sample functions of such a process are continuous if and only if the infinitely divisible distributions obtained in this way are Gaussian, so that the given process is essentially the one dimensional Brownian movement process reduced modulo $2 \pi$. In the Poisson 
kernel process these infinitely divisible distributions are of Cauchy type, of density $1 /\left[2 \pi\left(1+\eta^{2}\right)\right]$, aside from a scaling constant. Thus it follows from Lévy's general theorems that the sample functions of a separable Poisson process are almost all continuous, except for jumps, and these jumps actually occur. From the point of view of this paper, the Poisson kernel process can be generated as follows. Let $\{z(t), 0 \leqq t<\infty\}$ be a plane Brownian motion process with initial point the origin, and let $z_{1}(t)$ be the first point of the circle $C_{t}$ with center the origin and radius $t$ in which a Brownian path from the origin meets $C_{t}$. Then the process $\left\{\arg z_{1}(t), 0<t<\infty\right\}$ is a Poisson kernel process, aside from the fact that 0 is not in the parameter set. In fact we have remarked that a Brownian path, after its first meeting with $C_{s}$, proceeds like a Brownian path with an initial distribution, that of $z_{1}(s)$, on $C_{s}$, and, if $t>s$, this means that the distribution of $z_{1}(t)$, for $z_{1}(s)$ given and equal to $z_{1}$, is the harmonic measure on $C_{t}$ relative to $z_{1}$. This distribution, when described in terms of the angular coordinate on $C_{s}$ and $C_{t}$, is precisely the transition distribution of the Poisson kernel process. Note that this method of generating the Poisson kernel process makes the random variables of this process uniformly distributed on $[0,2 \pi)$. It is now obvious, even without Lévy's general theorems, that the sample functions of the process obtained in this way are almost all continuous except for jumps.

According to Theorem 6.3, with $D_{t}$ of that theorem identified with the interior of $C_{t}$ as just defined, and $u(w) \equiv w$, if $\{x(t), 0 \leqq t<\infty\}$ is a Poisson kernel process, and if $u$ is subharmonic [harmonic or regular] in $D_{R}$, then the process

$$
\left\{u\left[t e^{i x(t)}\right], 0 \leqq t<R\right\}
$$

is a semimartingale [martingale]. This fact is also easily proved directly. In fact, from the definition of the Poisson kernel process,

$$
E\left\{u\left[t e^{i x(t)}\right] \| x(s)\right\}=\int_{0}^{2 \pi} u\left(t e^{i \theta}\right) K[s, t, \theta-x(s)] d \theta .
$$

If $u$ is subharmonic [harmonic or regular] the right side is obviously greater than or equal [equal] to $u[x(s)]$.

The Poisson kernel process can be generalized as follows. Let $\left\{D_{t}, 0<t<\infty\right\}$ be a family of bounded plane domains, all containing the origin, with $D_{s} \subset D_{t}$ when $s<t$, and let $z_{1}(t)$ be the first point of the boundary $C_{t}$ of $D_{t}$ in which a Brownian path from the origin meets $C_{t}$. According to Theorem 6.3 , with $u(w) \equiv w$, the process $\left\{z_{1}(t), 0<t<\infty\right\}$ is a martingale, and in fact remains one if $z_{1}(0)$ is defined as 0 and if 0 is adjoined to the parameter interval. The sample functions of the $z_{1}(t)$ process are almost all continuous except for jumps. The $z_{1}(t)$ process is a Markov process whose transition probabilities are determined very simply. If $s<t$, and if $z_{1}(s)$ has the value $z_{1}$, then, if $z_{1} \in C_{t}, z_{1}(t)$ is to have this same value, but otherwise the 
(conditional) distribution of $z_{1}(t)$ is the harmonic measure on $C_{t}$ relative to $z_{1}$. If $u$ is subharmonic [harmonic or regular] in $D_{R}$, the process $\left\{u\left[z_{1}(t)\right]\right.$, $0 \leqq t<R\}$ is a semimartingale [martingale], except that 0 is to be omitted from the parameter set if $u(0)=-\infty$. The Poisson kernel process is simple because there is a simple one to one way in which $C_{t}$ can be mapped on the interval $[0,2 \pi)$ when $C_{t}$ is a circle concentric with the origin, which makes the transition probabilities have simple expressions.

We make one final remark on the methods of this paper before turning to an examination of the $N$-dimensional case. The methods have been based on the invariance of the semimartingale and martingale properties of a stochastic process under optional stopping and sampling, as developed in [7]. However the stopping and sampling used in this paper have all been of one very special type, based on the first meeting of a Brownian path with a closed set. Many of the results would hold for more complicated sampling or stopping rules, and have been stated in unnecessarily special form only to avoid complications unnecessary for the purposes of this paper.

7. Summary in the $N$-dimensional case $(N>2)$. In the following we outline the analogues of the results of the previous sections in the $N$-dimensional case, for $N>2$. The theory is somewhat simpler in this case, because the potential of a distribution is positive. If $z_{1}, z_{2}$ are points in $N$ dimensions, we write $\left|z_{1}-z_{2}\right|$ for the distance between them.

The discussion in $\$ 1$ needs no change, except that in the discussion of subharmonic functions the domains are now $N$-dimensional, and that averages over circle perimeters become averages over spherical surfaces. In the following, when only such trivial changes are involved to translate a result into $N$ dimensions, the result will be said to go over with only dimensionality changes.

Lemma 2.1 goes over with only dimensionality changes. In Lemma 2.2, the function $u^{*}$ satisfies (a) of the statement, but, instead of (b), $u^{*}-c|z|^{2-N}$ is now harmonic in an extended neighborhood of $\infty$, if defined properly at $\infty$. The proof is unchanged, except that $\log |z|$ is replaced by $-|z|^{2-N}$. (Here and in similar expressions below, the obvious infinite value of an expression is to be understood when it is undefined.)

Lemma 2.3 is somewhat simpler in $N>2$ dimensions. The function $v$ of that lemma is now given by

$$
v(z)=-\sum_{j=1}^{n} \frac{c_{j}}{\left|z-z_{j}\right|^{N-2}}, \quad c_{j}>0 .
$$

In part (b) of that lemma, if $D$ is not a neighborhood of $\infty$, $w$ can be defined at $\infty$ to be harmonic in an extended neighborhood of $\infty$. Otherwise there are only dimensionality changes.

No changes need be made in $\S 3$, because the discussion is given for $N \geqq 1$.

We shall discuss only subharmonic and harmonic functions in $N$-space, 
ignoring the obvious related results on functions of more than one complex variable and on hypercomplex functions. In the $N$-dimensional versions of the theorems of $\S 4$, the "regular" will thus have no counterpart.

Theorem 4.1 needs only dimensionality changes, including the replacement of (4.1) by

$$
\int_{0}^{\infty} u_{r} e^{-c r^{2} r^{N-1}} d r<\infty,
$$

where $u_{r}$ is the integral average of $|u|$ over a spherical surface with center the origin and radius $r$.

The corollary to Theorem 4.1 is true in $N>2$ dimensions if it is true in 2 dimensions. To give an independent proof, simply replace the function $u$ of the proof by

$$
-\left|z-z_{0}\right|^{2-N}-\left|z-z_{1}\right|^{2-N} \text {. }
$$

Lemma 4.2 is true in $N>2$ dimensions if the logarithm process of the lemma is replaced by the process

$$
\left\{-\left|z^{*}(t)-z_{1}\right|^{2-N}, 0 \leqq t<\infty\right\} .
$$

The proof requires only dimensionality changes, including an evaluation of the probability $p$ that a Brownian path from a point between two concentric spherical shells meets the outer shell before the inner one. If the initial point of the path is at distance $\rho$ from the center, and if the shells have radii $r, R$, with $r<\rho<R$, the probability $p$ is given by

$$
p=\left(\rho^{2-N}-r^{2-N}\right)\left(R^{2-N}-r^{2-N}\right)^{-1} .
$$

The proof follows that in the two-dimensional case.

The corollary to Lemma 4.2 is false in $N>2$ dimensions. It is replaced by a more delicate result below. The following related result is due to Kakutani [10]. The proof is included here to exhibit the methods developed.

TheOREM 7.1. If $N>2$, and if $z$ is arbitrary, almost every Brownian path from $z$ approaches $\infty$ when $t \rightarrow \infty$.

According to the $N$-dimensional version of Theorem 4.1, if $\{z(t)$, $0 \leqq t<\infty\}$ is a Brownian motion process in $N>2$ dimensions, the process

$$
\left\{-|z(t)|^{2-N}, 0<t<\infty\right\}
$$

is a semimartingale, and almost all sample functions of this process are obviously continuous. Since the random variables of the process are negative, it is known from the general theory of semimartingales [7] that almost all the sample functions have a finite limit when $t \rightarrow \infty$, and this is obviously impossible unless $|z(t)| \rightarrow \infty$ with probability 1 .

Theorem 4.3 goes over with only dimensionality changes, including a 
change of the analytic condition of the theorem to the condition that the integral in $\left(4.1^{\prime}\right)$ be convergent in some neighborhood of $\infty$. The corollary needs only dimensionality changes.

The potential induced by a charge $\mu$ distributed over a compact subset $A$ of a sphere $D$ of radius $R$, relative to the bounding surface $C$, is, in $N>2$ dimensions, given by

$$
v(z)=\int_{A}\left(\frac{1}{|z-w|^{N-2}}-\frac{R^{N-2}}{|w|^{N-2}\left|z-w^{\prime}\right|^{N-2}}\right) \mu(d w) .
$$

Here $w$ is the spherical image of $w$ in $C$. Then $v \geqq 0,-v$ is subharmonic in $D$, harmonic in $D-A$, with boundary value 0 on $C$. We can also take $R=\infty$ here, obtaining the potential of this charge relative to the point $\infty$, given by

$$
v(z)=\int_{A} \frac{1}{|z-w|^{N-2}} \mu(d w) .
$$

The general remarks on capacity made in $\$ 5$ need only dimensionality changes. Some of the theorems in $\S 5$ are however profoundly altered by the fact that in $N>2$ dimensions, as Brelot has shown [3], the point $\infty$ should be regarded as having positive capacity. Some of the proofs in $\$ 5$ are most easily carried through in $N>2$ dimensions using potentials relative to the point $\infty$ instead of relative to a circle.

Theorem 5.1 needs only dimensionality changes. Theorem 5.2 is false in $N>2$ dimensions, and in fact Theorem 7.1, together with the fact that almost no Brownian path passes through a preassigned point, implies Kakutani's theorem [10] that almost every Brownian path is nowhere dense, if $N>2$. Dvoretzky, Erdös, and Kakutani [8] have shown that almost every Brownian path segment determined by a finite parameter interval has positive capacity if $N=3$, zero capacity if $N>3$.

Theorem 5.3, due to Kakutani for $A$ compact, needs only dimensionality changes. If $D$ is taken as the whole finite space, so that $C$ is the point $\infty$, the probability of meeting $A$ before $C$ becomes the probability of ever meeting $A$.

Lemma 5.4 and Theorems 5.5, 5.6, 5.7 need only dimensionality changes.

$\S \S 6$ and 7 need only dimensionality changes, except that the point $\infty$ is not to be in $D$ in Theorem 6.2 when $N>2$. The basic theorems on radial boundary values of functions subharmonic in a sphere are due to Privalov [15].

\section{BIBLIOGRAPHY}

1. M. Brelot, Familles de Perron et problème de Dirichlet, Acta Litt. Sci. Szeged vol. 9 (1939) pp. 133-153.

2. - Points irréguliers et transformations continues en théorie du potentiel, J. Math. Pures Appl. vol. 19 (1940) pp. 319-337. 
3. - Sur le role du point à l'infini dans la théorie des fonctions harmoniques, Ann. Sci. Ecole Norm. vol. 61 (1944) pp. 301-332.

4. - Le problème de Dirichlet "ramifiê," Ann. Univ. Grenoble Sect. Sci. Math. Phys. (N.S.) vol. 22 (1946) pp. 167-200.

5. Henri Cartan, Théorie du potentiel newtonien; énergie, capacité, suites de potentiels, Bull. Soc. Math. France vol. 73 (1945) pp. 74-106.

6. - Théorie générale du balayage en potentiel newtonien, Ann. Univ. Grenoble (N.S.) Sect. Sci. Math. Phys. vol. 22 (1946) pp. 221-280.

7. J. L. Doob, Stochastic processes, New York, 1953.

8. A. Dvoretzky, P. Erdös, and S. Kakutani, Double points of paths of Brownian motion in n-space, Acta Sci. Math. Szeged vol. 12 (B) (1950) pp. 75-81.

9. B. Hostinsky, Sur une équation fonctionnelle considérée par Chapman et par Kolmogoroff, C. R. (Doklady) Acad. Sci. URSS (N.S.) vol. 2 (1934) pp. 393-397.

10. S. Kakutani, On Brownian motions in n-space, Proc. Imp. Acad. Tokyo vol. 20 (1944) pp. 648-652.

11. Two-dimensional Brownian motion and harmonic functions, Proc. Imp. Acad. Tokyo vol. 20 (1944) pp. 706-714.

12. P. Lévy, L'addition des variables aléatoires définies sur une circonférence, Bull. Soc. Math. France vol. 67 (1939) pp. 1-41.

13. - Le mouvement Brownien plan, Amer. J. Math. vol. 62 (1940) pp. 487-550.

14. J. E. Littlewood, Mathematical Notes (8); on functions subharmonic in a circle (II), Proc. London Math. Soc. (2) vol. 28 (1928) pp. 383-394.

15. I. Privalov, Boundary functions of the theory of harmonic and subharmonic functions in space, Rec. Math. (Mat. Sbornik) N.S. vol. 3 (45) (1938) pp. 3-25.

16. J. L. Snell, Applications of martingale system theorems, Trans. Amer. Math. Soc. vol. 73 (1952) pp. 293-312.

17. Elmer Tolsted, Limiting values of subharmonic functions, Proc. Amer. Math. Soc. vol. 1 (1950) pp. 636-647.

UNIVERSITY OF IlLINOIS,

URBana, IlL. 